\title{
What can the cold-induced transcriptomes of Arctic Brassicaceae tell us about the evolution of cold tolerance?
}

Siri Birkeland ${ }^{1,2}$, Tanja Slotte ${ }^{1,3}$, Anne K. Brysting4, A. Lovisa S. Gustafsson¹, Torgeir

R. Hvidsten², Christian Brochmann ${ }^{1}$, Michael D. Nowak ${ }^{1}$

${ }^{1}$ Natural History Museum, University of Oslo, Oslo, Norway ${ }^{2}$ Department of Chemistry, Biotechnology and Food Science, Norwegian University of Life Sciences, Ås, Norway 3Department of Ecology, Environment, and Plant Sciences, Science for Life Laboratory, Stockholm University, Stockholm, Sweden 4 Centre for Ecological and Evolutionary Synthesis, Department of Biosciences, University of Oslo, Oslo, Norway

Corresponding author: Siri Birkeland, birkeland.siri@gmail.com

\section{Abstract}

- By studying the molecular basis of cold response in plants adapted to some of the world's coldest biomes, we can gain insight into the evolution of cold tolerance - an important factor in determining plant distributions worldwide.

- Although cold tolerance in temperate plants have been extensively studied, little is known about the evolutionary changes needed to transition from temperate to the more extreme polar zones.

- Here, we conducted a time series experiment to examine the transcriptional responses of three Arctic Brassicaceae to low temperatures. RNA was sampled before onset of treatment, and after $3 \mathrm{~h}, 6 \mathrm{~h}$, and $24 \mathrm{~h}$ with $2{ }^{\circ} \mathrm{C}$. We identified sets of genes that were differentially expressed in response to cold and compared them between species, as well as to published data from the temperate Arabidopsis thaliana.

- We found that the cold response is highly species-specific. Among thousands of differentially expressed genes, $\sim 200$ genes were shared among the three Arctic species and $A$. thaliana, and only $\sim 100$ genes were specific to the three 
Arctic species alone. This pattern was also reflected in the functional comparison.

- Our results show that the cold response of Arctic plant species has mainly evolved independently, although it likely builds on a conserved basis found across Brassicaceae. The findings also confirm that highly polygenic traits, such as cold tolerance, may show less repeatable patterns of adaptation than traits involving only a few genes.

\section{Introduction}

Temperature is one of the most important factors determining plant distributions across the world, and only a few species have been able to occupy the cold biomes found towards the poles (Wiens and Donoghue 2004; Billings and Mooney 1968). Evolutionary history seems to play an important role in determining how plant cold tolerance is distributed globally, with tropical-to-temperate transitions being key events in plant evolution (Lancaster and Humphreys 2020). However, little is known about the evolutionary changes required for the transition from temperate to the more extreme polar zones. It is still unclear if the cold response of polar plant species is distinct from that of temperate relatives, and whether it may have evolved in a similar or convergent fashion because of the same extreme selection pressures. Polar environments exhibit lower year-round temperatures and shorter growing seasons (with up to 24 hours of daylight) than temperate environments, although some temperate-alpine environments share similar characteristics (Billings 1974). In the Arctic, the average temperature of the warmest summer month is not more than 10 ${ }^{\circ} \mathrm{C}$ (Elvebakk 1999), and the growing season can be as short as one month in the coldest areas (e.g. Jónsdóttir 2005). By studying how Arctic plants cope with low temperatures, we can gain insights into how plants acquire cold tolerance, and if there are general trends in plant adaptation to extreme polar environments.

Plants face many challenges upon the transition to colder environments. Low temperatures can affect nearly all aspects of plant cell biochemistry, and influence protein properties, photosynthesis reactions, and cell membrane fluidity (Shi, Ding, and Yang 2018). Ice formation comes with its own set of challenges and is in general deadly if ice forms within the cell (Körner 2003). Some plants can tolerate freezing of the apoplast (the space between the cells), but this may draw water out of the cell and lead to severe dehydration, as well as increase the level of salts and toxic solutes 
(Wisniewski et al. 2004; Steponkus 1984; Körner 2003; Wisniewski and Fuller 1999). In temperate environments (from where Arctic plants most likely are derived; Abbott and Brochmann 2003), plants prepare for predictable cold periods via cold acclimation, i.e. an increase in freezing tolerance in response to low non-freezing temperatures (Thomashow 2010). Exposing temperate plants to low temperatures typically results in complete reorganization of the transcriptome, ultimately leading to increased freezing tolerance (Kreps et al. 2002; Thomashow 2010; Kilian et al. 2007). The CBF transcription factors (C-repeat-Binding Factors) are among the main "regulatory hubs" of this cold response, and have been isolated in many different plant species (Shi, Ding, and Yang 2018; Park et al. 2015; Thomashow 2010). The CBFs are induced shortly after exposure to cold stress, and control the expression of $>100$ cold-regulated (COR) genes downstream (the CBF pathway; Park et al. 2015). Arabidopsis thaliana exhibits three of these cold-induced CBFs ( $C B F_{1}, C B F_{2}$ and $C B F_{3}$, also called $D R E B 1 b, D R E B 1 c$ and DREB1a; Jia et al. 2016), but the same genes are not always found in other species (Zhao et al. 2012). It is also becoming increasingly clear that the $\mathrm{CBF}$ regulon involves extensive co-regulation by other lesser-known transcription factors, and that the low temperature regulatory network is highly complex (Park et al. 2015). One could envision that polar plant species are in less need of a cold acclimation period as temperatures are low year-round (e.g., their transcriptomes could be less responsive to a drop in temperature), or that their cold response is somehow more complex (e.g., involving more genes) or more effective (e.g., faster, or involving fewer genes). There are surprisingly few in-depth studies on the cold-induced transcriptomes of polar plants, but Archambault and Strömvik (2011) studied Arctic Oxytropis, Wang et al. (2017) the temperate-subarctic Eutrema (Thellungiella) salsugineum, and Lee et al. (2013) the Antarctic Dechampsia antarctica. Although these studies give valuable species-specific information on cold response, they give limited insight into how polar plants differ from temperate relatives. In this study, we therefore perform a whole-transcriptome investigation of cold response in three plant species adapted to the high Arctic, and then compare their response to that of temperate relatives.

The focal species of this study, Cardamine bellidifolia, Cochlearia groenlandica and Draba nivalis, have independent Arctic origins (Carlsen et al. 2009; Jordon-Thaden et al. 2010; Koch 2012) and represent three of the main clades of Brassicaceae (clade A, B, and C; divergence time 30 Mya; Huang et al. 2016). 
They are ideal model species for studying cold response of Arctic plants for three main reasons: 1) all have their main distribution above the Arctic Circle, 2) they are found in all Arctic bioclimatic subzones (even in polar deserts; Elven et al. 2011), and 3) they belong to the plant family in which cold response has been most extensively studied, as it includes the model species $A$. thaliana and various economically important crop species (Kilian et al. 2007; Park et al. 2015; Shi, Ding, and Yang 2018). Furthermore, we recently found evidence of positive selection in genes associated with cold stress in all three species (Birkeland et al. 2020). Different genes seem to be under positive selection in each species, suggesting that they have independently adapted to the Arctic by modifying different components of similar stress response pathways (Birkeland et al. 2020). However, our previous study was limited to protein coding regions, and theory predicts that there could be a higher chance of convergence in their expression profiles (e.g. Stern 2013; Sackton et al. 2019). The reason is that mutations in cis-regulatory regions should have fewer pleiotropic effects than mutations in coding regions, as protein function may be affected only in a subset of the full expression domain (Gompel and Prud'homme 2009; Stern 2013). This is tied to the fact that a gene can have several cis-domains and that one cis-domain may bind several different transcription factors. To evaluate the degree of similarity in Arctic Brassicaceae expression profiles and to describe how Arctic cold-induced transcriptomes differ from those of temperate relatives, we therefore subjected these three species to a simulated summer cold shock and identified differentially expressed genes after $3 \mathrm{~h}, 6 \mathrm{~h}$ and $24 \mathrm{~h}$ with cold treatment. We aimed to i) characterize the cold-induced transcriptomes of $C$. bellidifolia, $C$. groenlandica and D. nivalis, ii) describe how their cold response differ from that of A. thaliana, and iii) identify potential convergent expression patterns in the three Arctic species.

\section{Material and methods}

\subsection{Plant material}

For each species, we sowed seeds from a single selfed parent derived from wild Arctic populations from Alaska (Supplementary table 1). Seeds were sown in six pots per species (several seeds per pot), of which four pots were used as biological replicates at each time point (see below). Because the plants were siblings derived from selfed 
parents, and because selfing is assumed to be the predominant mode of reproduction of these species in the wild (Brochmann and Steen 1999), we expected the replicates to be close to genetically identical. The plants were grown in a natural daylight room in the phytotron at the University of Oslo from $22^{\text {nd }}$ of March to $18^{\text {th }}$ of May 2018 with day temperature at $22{ }^{\circ} \mathrm{C}$ and night temperature at $18{ }^{\circ} \mathrm{C}$. Supplementary artificial light was given from 08:00-24:00 to mimic Arctic light conditions (400 W highintensity discharge lamps), and moisture was at $\sim 65 \% \mathrm{RH}$.

\subsection{Cold shock treatment}

Eight weeks after sowing, the plants were given a 24-hour cold shock to simulate a sudden drop in temperature during a typical Arctic summer. At 13:00 p.m. (to minimize correlation with circadian change), we transferred the pots from the $22{ }^{\circ} \mathrm{C}$ daylight room to a $2{ }^{\circ} \mathrm{C}$ cooling room with artificial light from $250 \mathrm{~W}$ high-intensity discharge lamps (140-160 $\mu \mathrm{mol} \mathrm{m} \mathrm{m}^{-2} \mathrm{~s}^{-1}$ measured at plant height). Leaf tissue was sampled at four time points; just before they were transferred (oh; control), and after $3 \mathrm{~h}, 6 \mathrm{~h}$, and $24 \mathrm{~h}$. We sampled all six pots for RNA extractions at each time point, but only used the four best RNA extracts per time point for sequencing (i.e., in terms of RNA quality and quantity). These constituted our four biological replicates per time point per species. RNA was immediately extracted from fresh, fully expanded leaves as described below. During the $24 \mathrm{~h}$ cold shock treatment, the plants were given supplementary artificial light to replicate the conditions in which they were sprouted (see above).

\subsection{RNA extraction and sequencing}

For extraction of total RNA, we used the Ambion RNAqueous Kit (Thermo Fisher Scientific, Waltham, USA), following the manufacturer's protocol for fresh plant tissue: $\sim 50$ mg leaf tissue was immediately ground in Lysis/Binding Solution together with 1 volume of Plant RNA Isolation Aid and consecutively extracted. The RNA quantity was measured with Broad Range RNA Kit on a Qubit v.2.o fluorometer (Life Technologies, Carlsbad, USA); RNA quality with an Experion Automated

Electrophoresis System Station (Bio-Rad Laboratories, Hercules, USA) and a Nandrop One spectrophotometer (Thermo Fisher Scientific, Waltham, USA). The Norwegian Sequencing Centre (www.sequencing.uio.no) prepared the libraries using the TruSeq protocol for stranded mRNA (Illumina, San Diego, USA) and performed 
the sequencing. Samples were indexed, pooled, and run on three lanes (16 samples/lane) on an Illumina HiSeq 3000 (Illumina, San Diego, USA), producing paired end reads with a default insert size of $350 \mathrm{bp}$ and read lengths of $150 \mathrm{bp}$. The raw reads were quality-checked with FastQC v.0.11.8 (Andrews 2010), and a single FastQC report per species was generated with MultiQC v.1.7 (Ewels et al. 2016).

\subsection{Transcriptome assembly and annotation}

As there were no available genome assemblies at the start of this study, we assembled a reference transcriptome de novo for each species using Trinity v.2.8.5 (Grabherr et al. 2011) based on all acquired RNA samples. Trinity was run with the integrated Trimmomatic option (Bolger, Lohse, and Usadel 2014), strand-specificity, and a minimum assembled contig length of $300 \mathrm{bp}$. The transcriptomes were filtered and annotated with EnTAP (Eukaryotic Non-Model Transcriptome Annotation Pipeline; Hart et al. 2020) in two rounds: first to apply the EnTAP filtering option on the raw transcriptome (in order to reduce inflated transcript estimates), and a second time to annotate the highest expressed isoform and filter out contaminants (used for the annotation of DEGs; see below). For expression filtering, an alignment file was generated with bowtie2 (Langmead and Salzberg 2012) in combination with RSEM (B. Li and Dewey 2011) using default options in the “align_and_estimate_abundance.pl" script provided with the Trinity software suite. Numbers of complete and fragmented BUSCOs (Benchmarking Universal SingleCopy Orthologs) in the filtered transcriptomes were estimated with BUSCO v4.0.6 (Simão et al. 2015). The filtered transcriptomes were used as the final reference in the differential expression analyses (see below).

\subsection{Differential expression analyses}

The Trimmomatic filtered reads were mapped to the reference transcriptomes using the alignment free mapper Salmon with a GC content bias correction (Patro et al. 2017). Genes that were differentially expressed after $3 \mathrm{~h}, 6 \mathrm{~h}$ and $24 \mathrm{~h}$ of cold treatment were identified with DESeq2 v.1.22.1 (Love, Huber, and Anders 2014), using a design formula controlling for the effect of pot number (design $=\sim$ pot number + time). This means that we tested for the effect of time with $2{ }^{\circ} \mathrm{C}$ treatment, while controlling for the individual effects of the sampled pots. A generalized linear model was fitted to each gene and a Wald test (Love, Huber, and Anders 2014) applied to test if the $3 \mathrm{~h}$, 
$6 \mathrm{~h}$ and $24 \mathrm{~h}$ model coefficients differed significantly from zero when contrasted to the oh model coefficient. A gene was considered as differentially expressed if the transcript level exhibited $\geq$ twofold change in response to the cold treatment at the different time points (log2 fold change threshold $=1$ ). We used a BenjaminiHochberg adjusted p-value (Benjamini and Hochberg 1995) to evaluate the significance of each differentially expressed gene (False Discovery Rate cutoff of alpha $=0.05)$. Heatmaps of the top 30 differentially expressed genes with the lowest false discovery rate were generated with the pheatmap package in R (Kolde 2019) using the regularized log function (rld) on original count data. The mean expression value of a gene was subtracted from each observation prior to heatmap generation.

\subsection{Comparison of DEG sets among Arctic species and A. thaliana}

To enable the comparison of DEGs among the Arctic species, and among the Arctic species and $A$. thaliana, we used already published data on differentially expressed genes in wild type $A$. thaliana in response to $24 \mathrm{~h}$ chilling treatment (Table $\mathrm{S} 1 \mathrm{in}$ Park et al. 2015). In this experiment, $A$. thaliana wild type plants were grown at $22{ }^{\circ} \mathrm{C}$ and constant illumination, then exposed to a $4{ }^{\circ} \mathrm{C}$ chilling treatment for $24 \mathrm{~h}$ (Ws-2; see Park et al. 2015 for details). Many of our analyses thus focus on the $24 \mathrm{~h}$ DEG sets. We used two different approaches to compare the $24 \mathrm{~h}$ DEG sets among species. First, we ran OrthoFinder v.2.3.12 (Emms and Kelly 2019) to identify sets of genes putatively descended from a single gene in the last common ancestor of all four species (orthogroups), using the assembled transcriptomes (filtered based on highest expressed isoform) and the Araport11 peptide file of $A$. thaliana downloaded from www.arabidopsis.org. This enabled us to compare orthogroup identity among DEG sets. Second, we used the BLASTP (protein-protein) search of BLAST+ v.2.9.0 (Camacho et al. 2009) to identify putative $A$. thaliana homologs in the three Arctic species, using the Araport11 peptide file as database and each of the Arctic transcriptome files as query (with e-value $<0.01$ and max target seqs $=1$ ). This second approach enabled us to compare gene identity and function more closely among species, but with the caveat that the $A$. thaliana homolog might not always be the true homolog or represent the same gene function in all species. The significance of the overlaps among differentially expressed orthogroups at $24 \mathrm{~h}$ were evaluated using the supertest function in SuperExactTest v.1.o.7 (M. Wang, Zhao, and Zhang 2015). We also visualized potential unique overlaps among differentially expressed 
orthogroups at $24 \mathrm{~h}$ and among differentially expressed $A$. thaliana homologs at $24 \mathrm{~h}$, using UpSetR v.1.4.o (Conway, Lex, and Gehlenborg 2017). To compare transcription factor composition, we annotated the $A$. thaliana 24 h DEG set with EnTAP (as above).

\subsection{Gene Set Enrichment Analyses}

To characterize sets of upregulated and downregulated genes further functionally, we performed gene ontology (GO) enrichment analyses within the Biological Process (BP), Cellular Component (CC) and Molecular Function (MF) domains for each species and time point. We used the Fisher's exact test in combination with the elim algorithm implemented in topGO 2.34.0 of Bioconductor to test for overrepresented GO-terms in each set of significantly upregulated or downregulated genes (Gentleman et al. 2004; Alexa, Rahnenfuhrer, and Lengauer 2006). The elim algorithm works by traversing the GO-graph bottom-up and discarding genes that already have been annotated to significant child terms, and is the recommended algorithm by the creators of topGO due to its simplicity (Alexa, Rahnenfuhrer, and Lengauer 2006). For the enrichment analyses, we used the gene annotations of the transcriptomes as background gene sets in each test (using the GO-annotations acquired with EnTAP). For A. thaliana, we used the org.At.tair.db R package v.3.7.o to annotate the $24 \mathrm{~h}$ DEG set of Park et al. (2015), and for creating a background gene set used in the enrichment tests (Carlson 2018). A GO-term was considered significantly enriched if $\mathrm{p}<0.05$. We did not correct for multiple testing as the enrichment-tests were not independent. Overlaps among enriched GO-terms in similar DEG sets (i.e., upregulated, and downregulated genes at similar time points) were compared among species using UpSetR as above.

\subsection{Comparison of DEGs with data set on positively selected genes}

Because we previously have identified convergent substitutions and tested for positive selection in the three focal species (see Birkeland et al. 2020 for details), we were able to check for potential overlaps between positively selected genes/convergent genes and the $24 \mathrm{~h}$ DEG sets. We blasted the newly assembled transcriptomes against the transcriptomes of the previous study using BLASTP with an e-value cutoff of $<0.01$ and max target seqs $=1$. 


\subsection{Gene co-expression network analyses}

To identify co-expressed gene modules, we performed weighted correlation network analysis for each species using the R package WGCNA (Langfelder and Horvath 2008). The gene expression matrix was prepared by first filtering out genes with consistent low counts (row sums $\leq 10$ ), and then applying a variance stabilizing transformation within DESeq2. We also filtered out genes with low expression variance by only maintaining genes with variance ranked above the 25 percentile in each data set. A gene adjacency matrix was constructed using default settings and raised to a soft thresholding power of 18 (signed network type). The soft thresholding power was chosen based on recommendations in the WGCNA FAQs, since a scalefree topology fit was reached only at very high values. This is not uncommon when a subset of the samples is globally different from the rest (for instance cold treated versus non-cold treated samples), which causes high correlation among large groups of genes. The adjacency matrix was translated into a Topological Overlap Matrix (TOM) and the corresponding dissimilarity measure. Co-expressed gene modules were then identified by using the resulting dissimilarity matrix as input in an average linkage hierarchical clustering analysis and pruning the dendrogram into modules using the Dynamic Tree-Cut algorithm (minimum module size of 30, deepSplit of 2, and pamRespectsDendro as FALSE; Langfelder et al. 2008). Close modules were merged based on the correlation of their eigengenes (correlation threshold of 0.75). We identified hub genes within each module as the genes with the top $10 \%$ eigengene-based connectivity (module membership). We also calculated the correlation between each module and a binary measure of cold (o/1) as well as a time-based measure of cold (o, 3, 6, and 24 hours), which was visualized in a heatmap. Gene set enrichment analyses of each module were performed with topGO as described above.

\section{Results}

\subsection{Transcriptome assemblies and Differentially Expressed Genes (DEGs)}

The three de novo assemblies contained $~ 22,000-24,000$ (Trinity) genes and were highly complete in terms of BUSCOs ( $>90 \%$ complete; Table 1 ). We identified a 
gradual increase in the number of DEGs with time at $2{ }^{\circ} \mathrm{C}$. About 700-1000 DEGs were identified after $3 \mathrm{~h}$, with varying rates of increase at $6 \mathrm{~h}(+33 \mathrm{DEGs}$ in $C$. bellidifolia, +283 DEGs in C. groenlandica, and +895 DEGs in D. nivalis), and with 2500-3000 DEGs identified at 24h (Table 2; Supplementary tables S2-4). Initially, most DEGs were upregulated, but after $24 \mathrm{~h}$ we found similar numbers of downregulated and upregulated genes in all species (Table 2). Most $3 \mathrm{~h}$ and $6 \mathrm{~h}$ DEGs were also found in the $24 \mathrm{~h}$ set, but many DEGs were also unique for each time point (Supplementary tables $\mathrm{S}_{5}-7$ ).

Based on orthogroup identity, most 24h DEGs seemed to be species-specific, but we found 212 differentially expressed orthogroups shared by the three Arctic species and $A$. thaliana, and 106 differentially expressed orthogroups shared by Arctic species but not by $A$. thaliana (Figure 1). These unique orthogroup intersections corresponded to overlaps of 195 and 119 genes based on $A$. thaliana homologs, respectively (Supplementary Fig. 1). Note that several genes from the same species occasionally can have the same orthogroup identity. We also found that all species (i.e. all species combinations) shared significant orthogroup overlaps in the 24h DEG sets (all p < 0.01 based on the supertest; Supplementary table 8). Thus, our main findings were that the shared portion of the cold response was bigger than expected by chance, and that more genes were shared by the three Arctic species and A. thaliana, than by the Arctic species alone.

Table 1. Statistics for de novo transcriptome assemblies

\begin{tabular}{|c|c|c|c|c|}
\hline $\begin{array}{l}\text { Species } \\
\text { (source) }\end{array}$ & $\begin{array}{l}\text { Total no. read } \\
\text { pairs }\end{array}$ & $\begin{array}{l}\text { No. of "genes" } \\
\text { in final } \\
\text { assembly } \\
\text { [raw assembly] }\end{array}$ & $\begin{array}{l}\text { No. of isoforms } \\
\text { in final assembly } \\
\text { [raw assembly] }\end{array}$ & $\begin{array}{c}\text { \% complete } \\
\text { BUSCOs in } \\
\text { final } \\
\text { assembly }\end{array}$ \\
\hline $\begin{array}{l}\text { Cardamine } \\
\text { bellidifolia }\end{array}$ & $\begin{array}{c}403,256,653 \\
\left(16 \text { samples }^{\mathrm{a}}\right)\end{array}$ & $\begin{array}{c}21,818 \\
{[42,151]}\end{array}$ & $\begin{array}{c}42,646 \\
{[98,419]}\end{array}$ & $93.8 \%$ \\
\hline $\begin{array}{l}\text { Cochlearia } \\
\text { groenlandica }\end{array}$ & $\begin{array}{c}389,172,001 \\
\left(16 \text { samples }^{\mathrm{a}}\right)\end{array}$ & $\begin{array}{c}22,396 \\
{[49,768]}\end{array}$ & $\begin{array}{c}40,639 \\
{[102,855]}\end{array}$ & $93.4 \%$ \\
\hline $\begin{array}{l}\text { Draba } \\
\text { nivalis }\end{array}$ & $\begin{array}{c}368,925,923 \\
\left(16 \text { samples }^{\mathrm{a}}\right)\end{array}$ & $\begin{array}{c}23,871 \\
{[52,096]}\end{array}$ & $\begin{array}{c}46,282 \\
{[109,658]}\end{array}$ & $92.6 \%$ \\
\hline
\end{tabular}

${ }^{\mathrm{a} F o u r}$ replicates at four time points $(0 \mathrm{~h}, 3 \mathrm{~h}, 6 \mathrm{~h}, 24 \mathrm{~h}),{ }^{\mathrm{b}}$ Corresponding to Trinity genes (or transcript clusters) 
Table 2. Number [percentage] of differentially expressed genes (DEGs) after $3 \mathrm{~h}, 6 \mathrm{~h}$ and 24h with $2^{\circ} \mathbf{C}$. (bold $=$ total number of DEGs, $\uparrow / \downarrow=$ upregulated/downregulated DEGs)

\begin{tabular}{lccc}
\hline & $\mathbf{3 h}$ & $\mathbf{6 h}$ & $\mathbf{2 4 h}$ \\
\hline $\begin{array}{l}\text { Cardamine } \\
\text { bellidifolia }\end{array}$ & $\mathbf{1 0 1 2}$ & $\mathbf{1 0 4 5}$ & $\mathbf{2 5 2 0}$ \\
& $857 \uparrow, 155 \downarrow$ & $877 \uparrow, 168 \downarrow$ & $(1301 \uparrow, 1219 \downarrow)$ \\
Cochlearia & {$[85 \% \uparrow, 15 \% \downarrow]$} & {$[84 \% \uparrow, 16 \% \downarrow]$} & {$[52 \% \uparrow, 48 \% \downarrow]$} \\
groenlandica & $521 \uparrow, 212 \downarrow$ & $(636 \uparrow, 380 \downarrow)$ & $(1534 \uparrow, 1476 \downarrow)$ \\
& {$[71 \% \uparrow, 29 \% \downarrow]$} & {$[63 \% \uparrow, 37 \% \downarrow]$} & {$[51 \% \uparrow, 49 \% \downarrow]$} \\
Draba & $\mathbf{7 3 3}$ & $\mathbf{1 0 1 6}$ & $\mathbf{3 0 1 0}$ \\
nivalis & $(505 \uparrow, 183 \downarrow)$ & $(998), 585 \downarrow)$ & $(1484 \uparrow, 1355 \downarrow)$ \\
& {$[73 \% \uparrow, 27 \% \downarrow]$} & {$[63 \% \uparrow, 37 \% \downarrow]$} & {$[52 \% \uparrow, 48 \% \downarrow]$} \\
\hline
\end{tabular}

\subsection{Comparison of DEGs among species}

\subsubsection{Transcription factors in Arctic species}

Transcription factors, the genes potentially orchestrating the cold response, made up 9-14\% of all Arctic DEGs at all times (Table 3, Supplementary tables 2-4). AP2/ERF (Figure 2) was the most common family of transcription factors based on the $24 \mathrm{~h}$ DEG set; known to include important candidates for cold regulation such as CBFs/DREBs and RAVs.

Among the transcription factors in the AP2/ERF family were $C B F 1(D R E B 1 B)$ and $C B F_{4}(D R E B 1 D)$, which were upregulated upon cold treatment in all species. Other upregulated AP2/ERFs included DREB2A (at all time points in $C$. groenlandica, and at $24 \mathrm{~h}$ in C. bellidifolia), $D R E B 2 C$ (at all time points in $C$. bellidifolia and D. nivalis), DREB3 (at all time points in C. groenlandica and $D$. nivalis), and $R A V 1$ (found to be upregulated at $6 \mathrm{~h}$ and $24 \mathrm{~h}$ in C. bellidifolia).

Other common transcription factors in all three species were those containing SANT/Myb domains, MYC-type basic helix-loop-helix (bHLH) domains, basicleucine zipper domains, and NAC domains (Figure 2). Many of these transcription factors were among the DEGs shared only by Arctic species based on the $A$. thaliana homolog (for instance REVEILLE 2, RAP2.1O, RAP2.2, PCL1, and HY5; Supplementary table 9). 


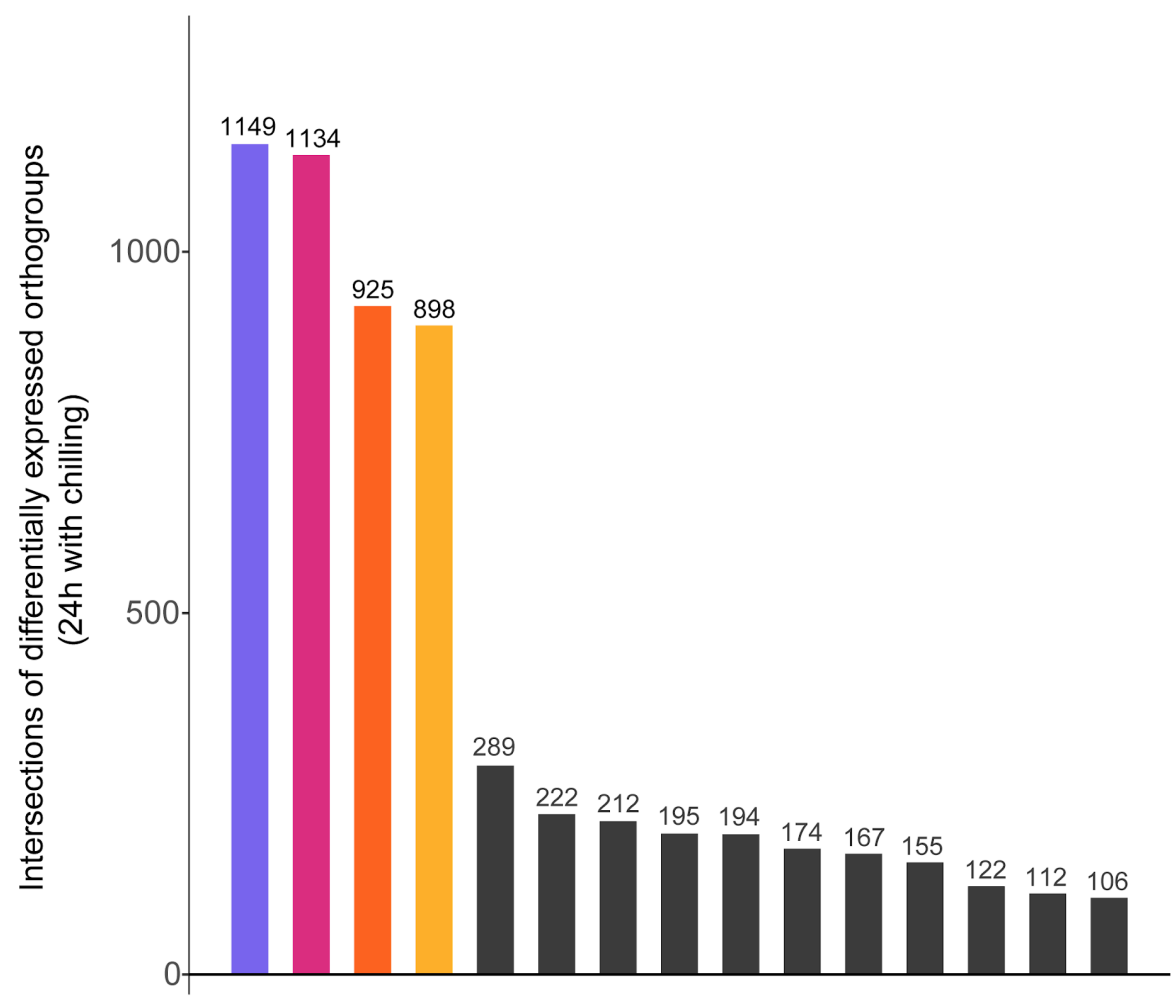

C. bellidifolia

D. nivalis

C. groenlandica

A. thaliana

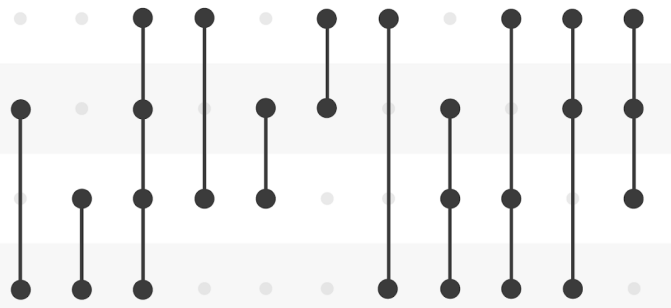

$2500200015001000500 \quad 0$

No. diff. expr. orthogroups per species

Figure 1. UpSet plot of differentially expressed orthogroups. The plot in the left corner shows total numbers of differentially expressed orthogroups, and the main plot shows the number of unique differentially expressed orthogroups, followed by orthogroups intersections/overlaps between species (connected dots).

Table 3. Number of differentially expressed genes (DEGs) annotated with "regulation of transcription" (GO:0006355) after $3 \mathrm{~h}, 6 \mathrm{~h}$ and $24 \mathrm{~h}$ with $2^{\circ} \mathrm{C}$. The percentage of the total DEG set is given in parentheses.

\begin{tabular}{lccc}
\hline & $\mathbf{3 h}$ & $\mathbf{6 h}$ & $\mathbf{2 4 h}$ \\
\hline Cardamine bellidifolia & $\mathbf{1 2 3}$ & $\mathbf{1 3 9}$ & $\mathbf{2 6 6}$ \\
& $(12 \%)$ & $(13 \%)$ & $(11 \%)$ \\
Cochlearia groenlandica & $\mathbf{1 0 6}$ & $\mathbf{1 4 3}$ & $\mathbf{3 4 4}$ \\
& $(14 \%)$ & $(14 \%)$ & $(11 \%)$ \\
Draba nivalis & $\mathbf{9 2}$ & $\mathbf{1 8 0}$ & $\mathbf{2 6 0}$ \\
& $(13 \%)$ & $(11 \%)$ & $(9 \%)$ \\
\hline
\end{tabular}


A.

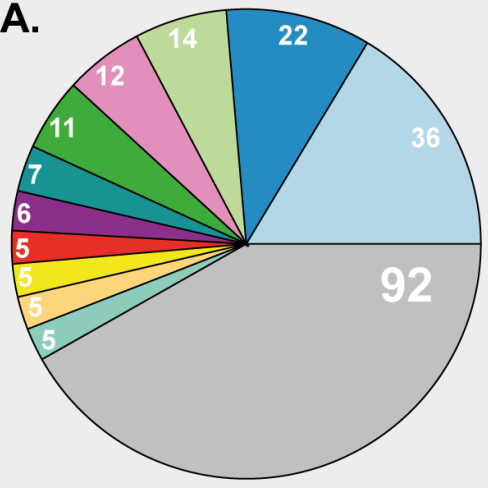

B.

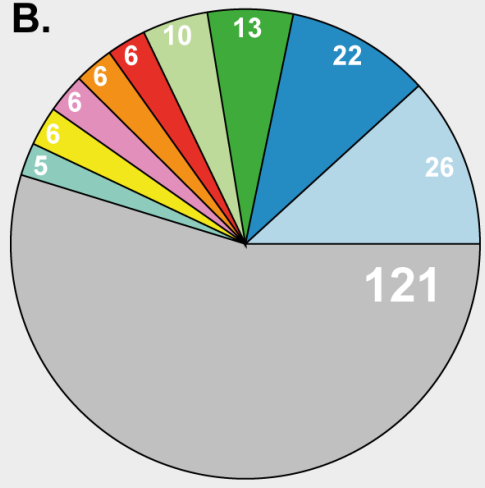

SANT/Myb domain

AP2/ERF domain

Myc-type, basic helix-loop-helix (bHLH) domain

NAC domain

Heat shock factor (HSF)-type, DNA-binding

WRKY domain

Transcription termination factor, mitochondrial/chloroplastic

Basic-leucine zipper domain

Signal transduction response regulator, receiver domain

Transcription factor GRAS

AUX/IAA domain

Transcription factor TCP subgroup

B-box-type zinc finger

Zinc finger, Dof-type

HD-ZIP protein, $\mathrm{N}$-terminal

CCT domain

Other/fewer than 5 of each
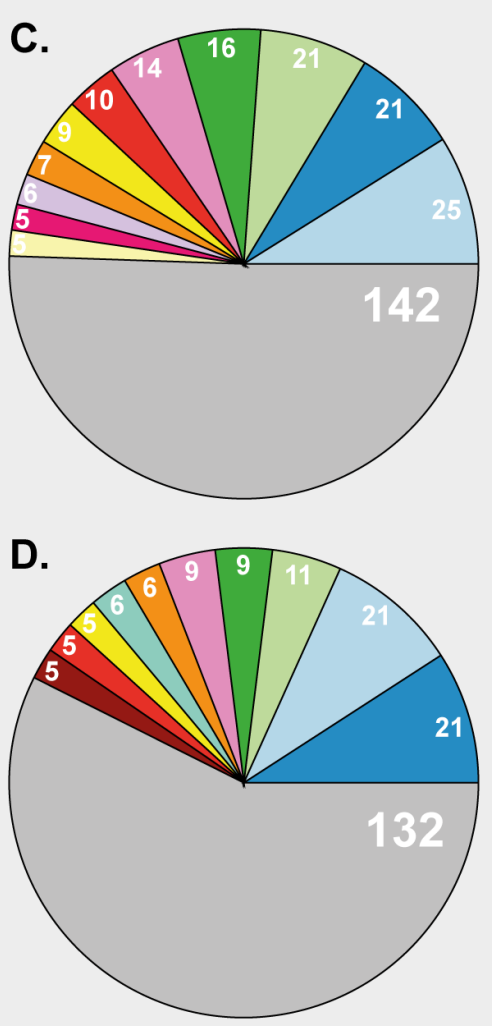

A. C. bellidifolia

B. D. nivalis

C. C. groenlandica

D. A. thaliana

Figure 2. InterPro domains in 24h DEGs annotated with "regulation of transcription" (GO:0006355). Transcription factors that did not have InterPro domain information are not included.

\subsubsection{Other shared cold regulated genes in Arctic species}

The gene overlaps based on A. thaliana homologs were used to further investigate cold regulated genes that were common in the cold response of all Arctic species at 24h (Supplementary tables 9-13). Among the 119 uniquely shared Arctic DEGs, 109 shared similar expression patterns in all species (74 upregulated and 35 downregulated; Supplementary figure 2). The upregulated DEGs might be especially important in acquiring freezing tolerance, and included many transcription factors (15, cf. above), but also genes annotated with e.g., response to karrikin (7), circadian rhythm (6), flavonoid biosynthetic process (5), and proline transport (4;

Supplementary table 9). Other upregulated DEGs shared by the Arctic species included e.g., MAPK7, MAPKKK14, MAPKKK18, SUCROSE SYNTHASE 3, RAB18, and a Late Embryogenesis Abundant gene (LEA; Supplementary table 9). Several of 
the uniquely shared DEGs could be found in the heatmaps of the top 30 differentially expressed genes with the lowest false discovery rate (Figure 3).

\subsubsection{Shared cold regulated genes among Arctic species and A. thaliana} Among the 195 DEGs shared by Arctic species and A. thaliana, 188 shared similar expression patterns in all species (Supplementary table 10). Among the 122 shared upregulated DEGs (Supplementary figure 2) were several genes annotated with e.g., response to cold (23), response to salt stress (20), response to abscisic acid (16), response to water deprivation (14), and flavonoid biosynthetic process (13; (Supplementary table 10). The shared gene list included many known cold induced genes like LEA14, COR78, COR15B, TCF1, COR27 and COR28 (Supplementary table 10). There were also several shared genes related to Abscisic acid and ethylene regulated pathways, like $A B F 1, A I T R 5, E R T 2$, and ERFO43 (Supplementary table 10). Several of the DEGs shared between A. thaliana and the three Arctic species could also be found in the heatmaps of the top 30 differentially expressed genes with the lowest false discovery rate (Figure 3).

\subsection{Functional characterization of DEG sets (GO-enrichment)}

Most significantly enriched GO-terms within the BP, CC, and MF domains were species-specific (Table 4, Supplementary tables 14-22). However, as many as 27 of the same GO-terms (BP and MF) were significantly enriched in the Arctic upregulated DEG sets at 24h (Supplementary table 23). Most of these (22) were also found in $A$. thaliana. Among the GO-terms shared by A. thaliana and the Arctic species were many BP terms like "response to cold", and "response to heat" (Figure 4-5). Other shared BP terms included those associated with cold and freezing like "response to salt stress", "response to water deprivation", "response to oxidative stress", and "response to cadmium ion" (Figure 5). We also observed a shared overrepresentation of genes associated with the hormones abscisic acid, and ethylene, and the substance karrikin, as well as genes associated with possible cryoprotectants like flavonoids and sucrose (BP, Figure 5). Among the GO-terms uniquely found in Arctic 24h upregulated DEG sets were e.g., "spermidine biosynthetic process" (BP) and "arginine decarboxylase activity" (MF). The 24h downregulated DEGs were enriched for genes associated with growth related GO-terms such as "phototropism" (BP; all species) and "auxin-activated signaling pathway" (BP; only Arctic species) 
bioRxiv preprint doi: https://doi org/10.1101/2021.12 04.471218. this version posted December 5, 2021. The copyright holder for this preprint (which was not certified by peer review) is the author/funder, who has granted bioRxiv a license to display the preprint in perpetuity. It is made available under aCC-BY-NC 4.0 International license.

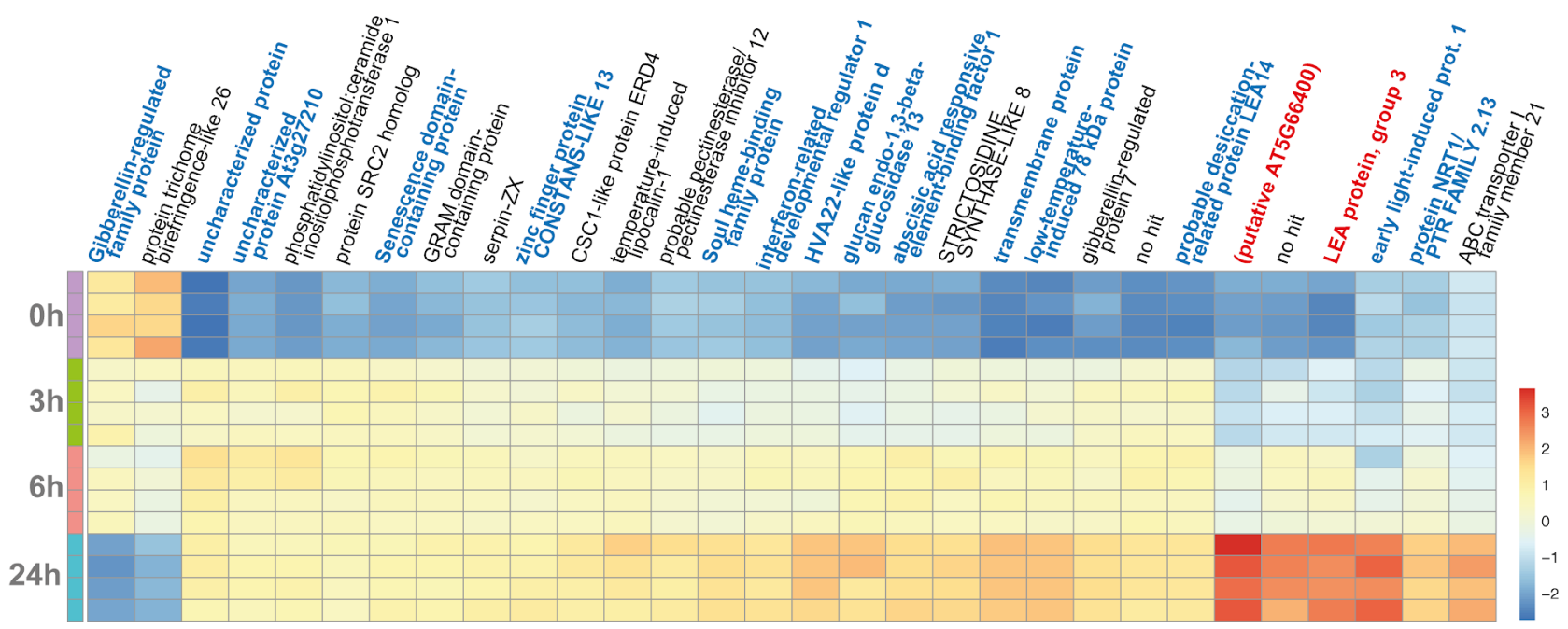

A. Cardamine bellidifolia

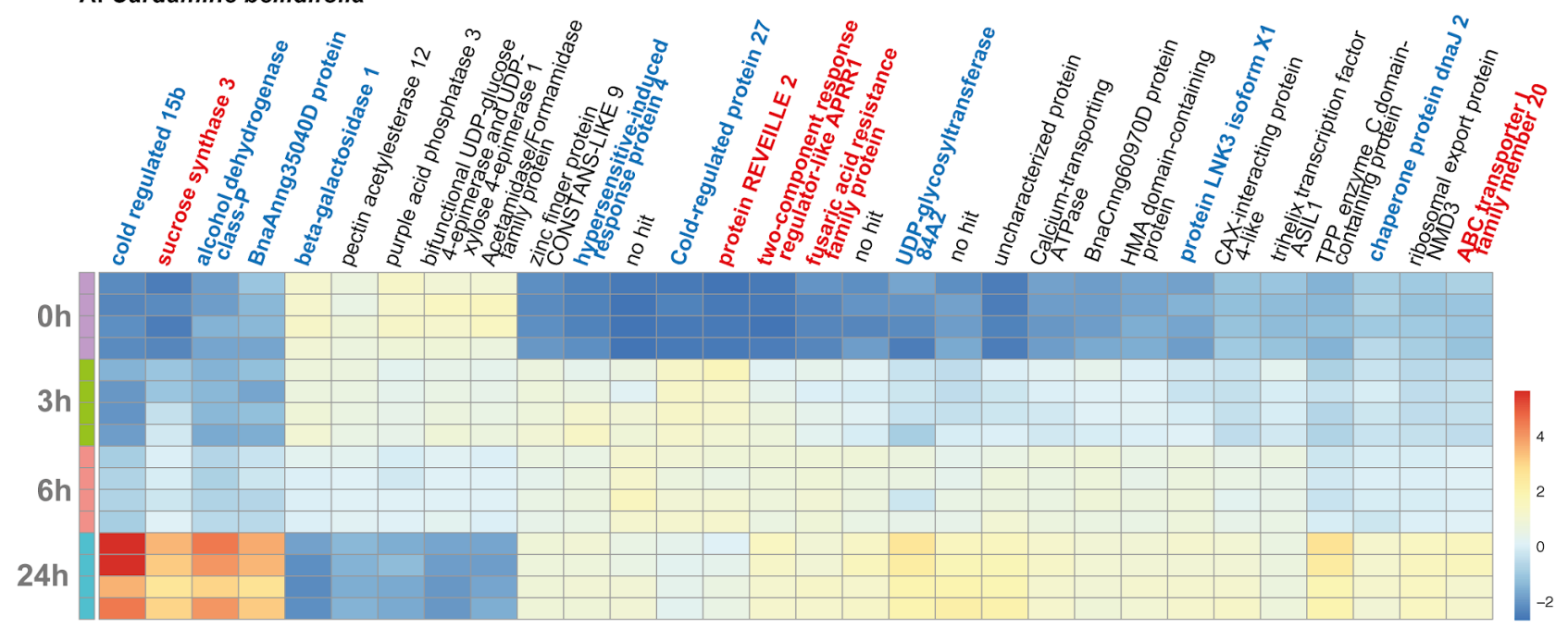

B. Draba nivalis

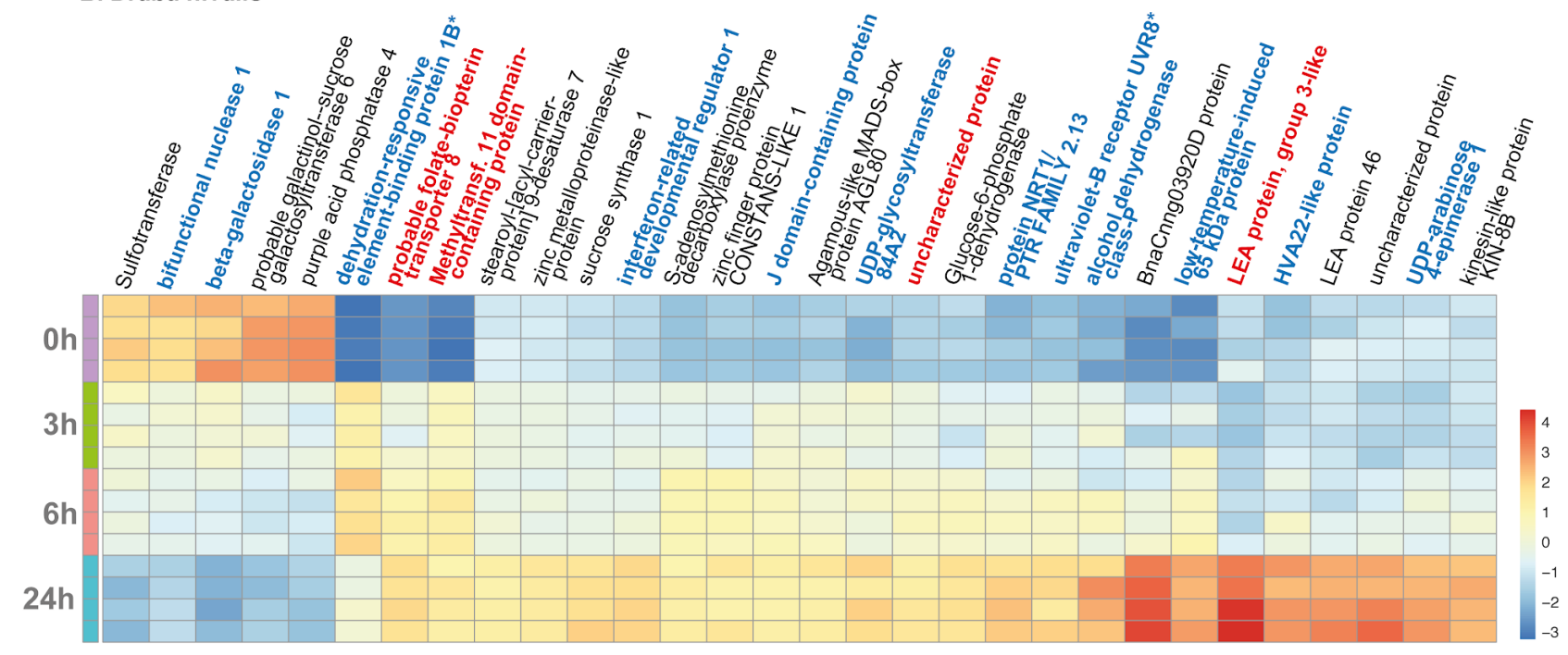

C. Cochlearia groenlandica

Figure 3. Heatmaps of the most significantly differentially expressed genes (DEGs): A) Cardamine bellidifolia, B) Draba nivalis, and C) Cochlearia groenlandica. Color scale $=\log 2$ transformed counts. Each row corresponds to a replicate, and there are four replicates at each time point (0h, 3h, 6h, 24h). Gene names in bold and blue = Found as 24h DEG in Arabidopsis thaliana and all Arctic species (based on $A$. thaliana homologs), gene names in bold and red = Found as 24h DEG only in Arctic species (based on $A$. thaliana homologs). *dehydration-responsive element-binding protein $1 \mathrm{~B}=\mathrm{DREB} 1 \mathrm{~b} / \mathrm{CBF} 1$, ultraviolet-B receptor UVR8 $=$ TCF1. 
Table 4. Numbers of significantly enriched GO-terms $(p<0.05)$ in differentially expressed genes (DEGs) after $3 \mathrm{~h}, 6 \mathrm{~h}$ and $24 \mathrm{~h}$ at $2{ }^{\circ} \mathrm{C}$ when applying the elim algorithm. Abbreviations: $\uparrow=$ Upregulated DEG set, $\downarrow=$ Downregulated DEG set, Biological Process $=$ $\mathrm{BP}$, Cellular Component $=\mathrm{CC}$, Molecular Function $=\mathrm{MF}$ domains.

\begin{tabular}{|c|c|c|c|c|c|}
\hline & & C. bellidifolia & C. groenlandica & D. nivalis & Shared* \\
\hline \multirow[t]{2}{*}{$\begin{array}{l}3 \mathrm{~h} \\
* * * 6\end{array}$} & $\uparrow$ & $\begin{array}{c}109 \mathrm{BP}, 12 \mathrm{CC}, \\
40 \mathrm{MF}\end{array}$ & $\begin{array}{c}101 \mathrm{BP}, 26 \mathrm{CC} \\
42 \mathrm{MF}\end{array}$ & $\begin{array}{c}102 \mathrm{BP}, 4 \mathrm{CC}, \\
38 \mathrm{MF}\end{array}$ & $\begin{array}{c}11 \mathrm{BP}, 1 \mathrm{CC}, \\
3 \mathrm{MF}\end{array}$ \\
\hline & $\downarrow$ & $\begin{array}{c}44 \mathrm{BP}, 7 \mathrm{CC}, \\
26 \mathrm{MF}\end{array}$ & $\begin{array}{c}61 \mathrm{BP}, 14 \mathrm{CC}, \\
34 \mathrm{MF}\end{array}$ & $\begin{array}{c}101 \mathrm{BP}, 1 \mathrm{CC}, \\
44 \mathrm{MF}\end{array}$ & $\begin{array}{c}0 \mathrm{BP}, 0 \mathrm{CC} \\
0 \mathrm{MF}\end{array}$ \\
\hline \multirow[t]{2}{*}{ 6h } & $\uparrow$ & $\begin{array}{c}127 \mathrm{BP}, 13 \mathrm{CC}, \\
33 \mathrm{MF}\end{array}$ & $\begin{array}{c}108 \mathrm{BP}, 5 \mathrm{CC}, \\
37 \mathrm{MF}\end{array}$ & $\begin{array}{c}120 \mathrm{BP}, 9 \mathrm{CC}, \\
44 \mathrm{MF}\end{array}$ & $\begin{array}{c}16 \mathrm{BP}, 1 \mathrm{CC}, \\
4 \mathrm{MF}\end{array}$ \\
\hline & $\downarrow$ & $\begin{array}{l}51 \mathrm{BP}, 4 \mathrm{CC}, \\
25 \mathrm{MF}\end{array}$ & $\begin{array}{c}73 \mathrm{BP}, 8 \mathrm{CC}, \\
37 \mathrm{MF}\end{array}$ & $\begin{array}{c}121 \mathrm{BP}, 8 \mathrm{CC} \\
45 \mathrm{MF}\end{array}$ & $\begin{array}{c}1 \mathrm{BP}, 0 \mathrm{CC} \\
0 \mathrm{MF}\end{array}$ \\
\hline \multirow[t]{2}{*}{$\begin{array}{l}24 \mathrm{~h} \\
*\end{array}$} & $\uparrow$ & $\begin{array}{c}156 \mathrm{BP}, 22 \mathrm{CC}, \\
62 \mathrm{MF}\end{array}$ & $\begin{array}{c}172 \mathrm{BP}, 30 \mathrm{CC}, \\
76 \mathrm{MF}\end{array}$ & $\begin{array}{c}139 \mathrm{BP}, 24 \mathrm{CC}, \\
82 \mathrm{MF}\end{array}$ & $\begin{array}{c}22 \mathrm{BP}, 0 \mathrm{CC}, \\
5 \mathrm{MF}\end{array}$ \\
\hline & $\downarrow$ & $\begin{array}{c}138 \mathrm{BP}, 10 \mathrm{CC}, \\
78 \mathrm{MF}\end{array}$ & $\begin{array}{c}108 \mathrm{BP}, 11 \mathrm{CC}, \\
60 \mathrm{MF}\end{array}$ & $\begin{array}{c}136 \mathrm{BP}, 25 \mathrm{CC}, \\
62 \mathrm{MF}\end{array}$ & $\begin{array}{c}7 \mathrm{BP}, 4 \mathrm{CC}, \\
0 \mathrm{MF}\end{array}$ \\
\hline
\end{tabular}

\subsection{Note on positively selected/convergent cold-responsive genes}

We identified several positively selected and convergent genes in the $24 \mathrm{~h}$ DEG sets of the Arctic species (Table 5-6; Supplementary tables 24-25; (Birkeland et al. 2020). Upregulated positively selected genes (PSGs) included genes such as COR15B and CSDP1 in D. nivalis, LEA4-5 in C. groenlandica, and a highly upregulated transmembrane protein (putative homolog of $A$. thaliana AT1G16850) in $C$. bellidifolia. We found that a gene with convergent substitutions in all Arctic species, EMB2742, was upregulated in all species (and in A. thaliana). Some convergent genes showed different expression patterns depending on the species. For instance, $C A T 2$ was downregulated in D. nivalis and upregulated in C. groenlandica. This gene has previously been found to contain convergent substitutions in $D$. nivalis and $C$. groenlandica, and to be under positive selection in D. nivalis. The low temperature responsive transcription factor $R A V 1$ has previously been found to be under positive selection in C. groenlandica, but was not differentially expressed in this species. We also note that MAPKKK14, (previously found to contain convergent substitutions in 
the Arctic species) had blast hits in several different MAPKKKs in this study. Its status as a convergent gene is therefore uncertain.
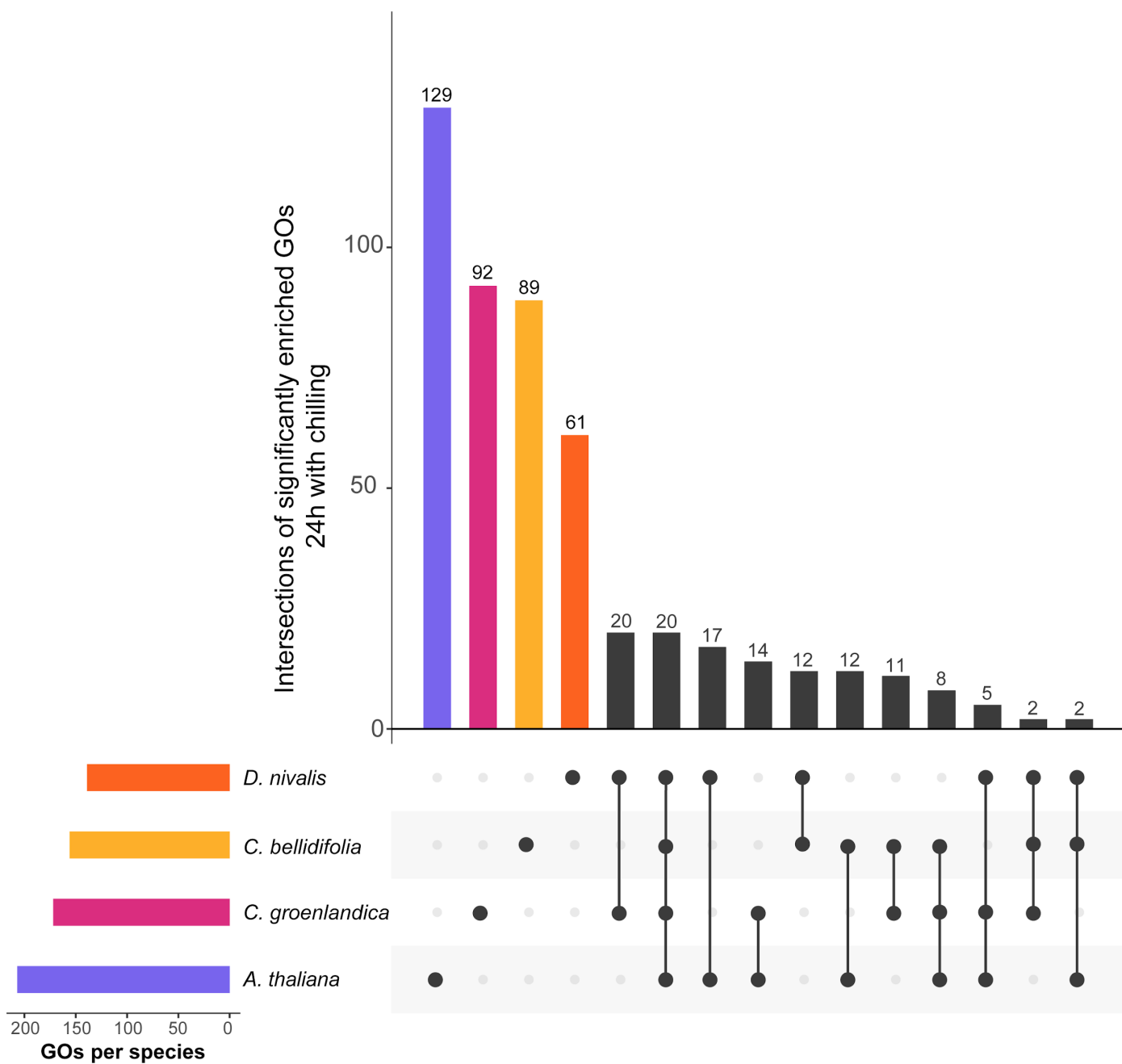

Figure 4. UpSet plot of overrepresented GO-terms in the 24h upregulated gene sets (Biological Process domain only). The plot in the left corner shows total numbers of significantly enriched GO-terms, and the main plot shows the number of unique significantly enriched GO-terms, followed by GO-term intersections/overlaps between species (connected dots).

Table 5. 24h DEGs under positive selection based on Birkeland et al. (2020).

PSG $=$ Positively selected gene

Total number of

PSGs Birkeland et al. (2020)

\section{PSGs among all}

DEGs, 24h

\section{PSGs among} upregulated DEGs, 24h
PSGs among downregulated DEGs, 24h

\begin{tabular}{lllll} 
C. bellidifolia & 201 & 26 & 14 & 12 \\
C. groenlandica & 159 & 23 & 12 & 11 \\
D. nivalis & 360 & 46 & 24 & 22 \\
\hline
\end{tabular}


Table 6. 24h DEGs with convergent substitutions based on Birkeland et al. (2020).

$\mathrm{Cb}=$ Cardamine bellidifolia $24 \mathrm{~h}$ DEG set, $\mathrm{Cg}=$ Cochlearia groenlandica $24 \mathrm{~h}$ DEG set, $\mathrm{Dn}=$ Draba nivalis $24 \mathrm{~h}$ DEG set

\begin{tabular}{ccc}
$\begin{array}{c}\text { Total number of } \\
\text { convergent }\end{array}$ & $\begin{array}{c}\text { Convergent } \\
\text { genes among all } \\
\text { genes Birkeland } \\
\text { et al. (2020) }\end{array}$ & $\begin{array}{c}\text { DEGs, 24h } \\
\text { genes among } \\
\text { upregulated } \\
\text { DEGs, 24h }\end{array}$ \\
\hline
\end{tabular}

Convergent
genes among
downregulated
DEGs, 24h

\begin{tabular}{lcccc}
\hline C. bellidifolia, & 58 & $17 \mathrm{Cb}$ & $9 \mathrm{Cb}$ & $8 \mathrm{Cb}$ \\
C. groenlandica & $14 \mathrm{Cg}$ & $12 \mathrm{Cg}$ & $2 \mathrm{Cg}$ \\
C. groenlandica, & 33 & $8 \mathrm{Cg}$ & $5 \mathrm{Cg}$ & $3 \mathrm{Cg}$ \\
D. nivalis & $8 \mathrm{Dn}$ & $2 \mathrm{Dn}$ & $6 \mathrm{Dn}$ \\
D. nivalis, & & & $13 \mathrm{Dn}$ & $6 \mathrm{Dn}$ \\
C. bellidifolia & 126 & $20 \mathrm{Cb}$ & $17 \mathrm{Cb}$ & $3 \mathrm{Cb}$ \\
\hline
\end{tabular}

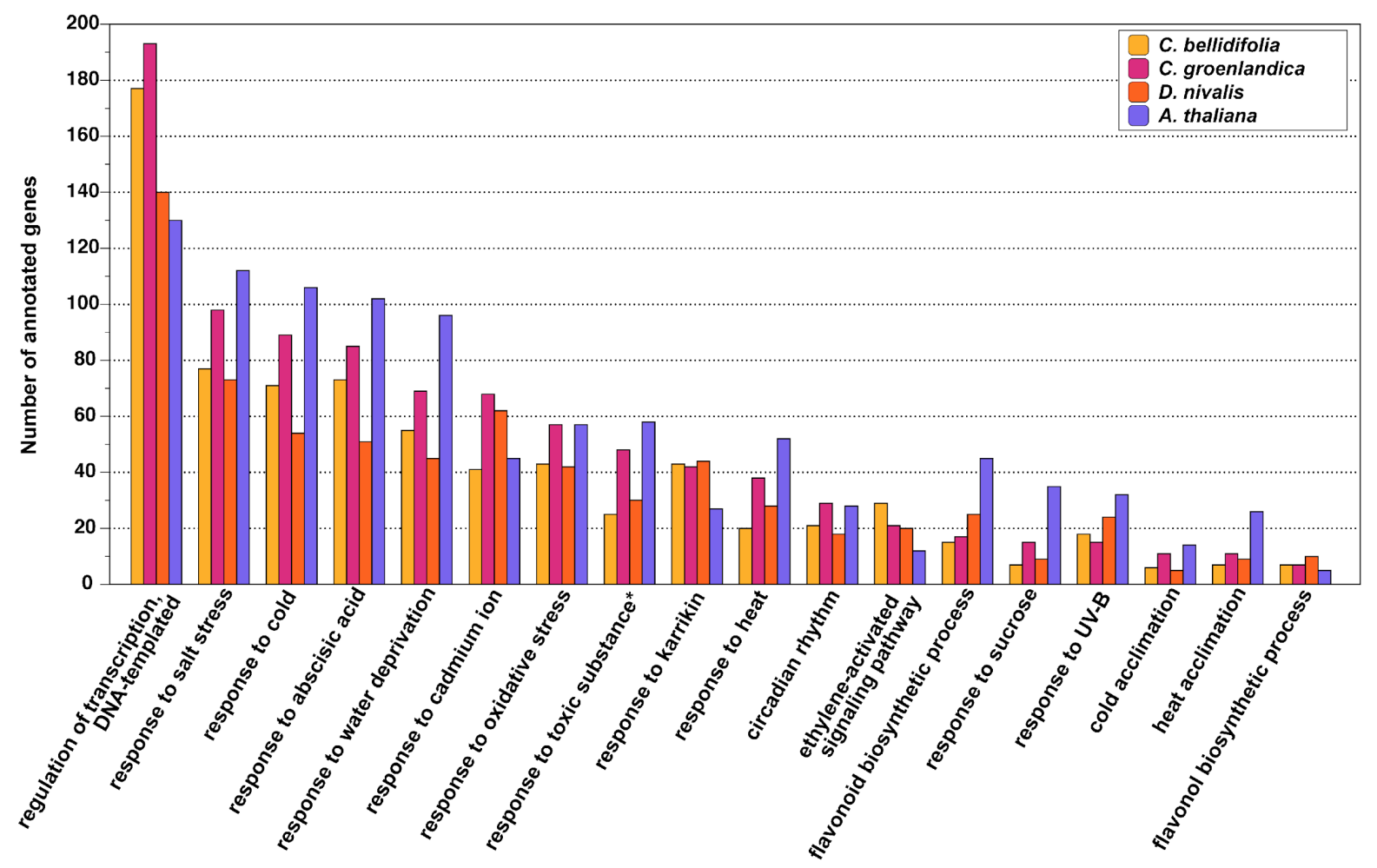

Figure 5. Barchart of genes annotated with Gene Ontology (GO) terms found to be significantly enriched in the $24 \mathrm{~h}$ upregulated gene set of the three Arctic species. There were 22 overlapping GO-terms among the Arctic 24h upregulated DEG sets, and 20 of these overlapped with $A$. thaliana. Only terms with at least 10 annotated genes are shown. *Not significantly enriched in $A$. thaliana. 


\subsection{Gene co-expression modules}

The gene co-expression network analyses resulted in 13 co-expressed modules in $C$. bellidifolia, 23 co-expressed modules in C. groenlandica and 14 co-expressed modules in D. nivalis (after module merging; Figure 6; Supplementary figure 3). At least one module in each species stood out as highly positively correlated with the binary measure of cold temperature (i.e., Pearson correlation coefficient $>0.90$ and $p$ $<$ 0.001), but often several modules in each species were positively correlated with this measure, and with $3 \mathrm{~h}, 6 \mathrm{~h}$ and $24 \mathrm{~h}$ of cold.

Focusing on the highest positively correlated module in relation to the binary measure of cold in each species (lightyellow in $C$. bellidifolia, lightcyan in $C$. groenlandica, and darkorange2 in D. nivalis, Figure 6), we found that 16 significantly enriched GO-terms overlapped between the modules in question (domain BP, CC, MF; Table 7). However, most GO-term enrichment results were module or speciesspecific (Supplementary tables 26-34). The three cold correlated modules had slightly different hub-genes (genes with high kME), but some general patterns were found (Supplementary tables 35-37): $M A P K 7$ (a gene found only in the 24h DEG sets of all Arctic species) was among the hub-genes of $C$. bellidifolia and D. nivalis, CONSTANS-like 4 was among the hub genes of C. groenlandica and D. nivalis, and CIPK4 had high kME in all species and was a hub gene in $D$. nivalis. In addition, several related genes like REVEILLE1, 2 and 6, and CONSTANS-like 4, 9, 10 and 13 had high $\mathrm{kmE}$ in all species, but the exact genes differed from species to species. We also noticed several well-known cold regulated genes among the hub genes in these modules like, for instance, $C O R 78$ and $C O R 47$ in the lightyellow module of $C$. bellidifolia, $C B F 1$ in the lightcyan module of $C$. groenlandica and COR27 in the darkorange2 module of $D$. nivalis. (Note that the genes orchestrating each module may have different expression patterns and that it is possible that they do not cluster with the module they are regulating). 


\begin{tabular}{|c|c|c|c|c|c|c|c|c|c|c|}
\hline (a) & Cardamine & bellidifolic & & & & & & & & \\
\hline brown4 [40] & -0.43 & $(0.09)$ & 0.064 & $(0.8)$ & 0.49 & $(0.05)$ & $-0.12(0.7)$ & 0.43 & $(0.09)$ & \\
\hline darkmagenta [2961] & -0.17 & $(0.5)$ & -0.45 & $(0.08)$ & -0.35 & $(0.2)$ & $0.98(6 e-11)$ & 0.17 & (0.5) & \\
\hline lightyellow [3940] & -0.94 & $(4 \mathrm{e}-08)$ & 0.093 & $(0.7)$ & 0.26 & $(0.3)$ & $0.59(0.02)$ & 0.94 & $(4 e-08)$ & \\
\hline darkgreen [4440] & 0.87 & $(1 e-05)$ & -0.0023 & $(1)$ & -0.14 & $(0.6)$ & $-0.73(0.001)$ & -0.87 & $(1 e-05)$ & 0.5 \\
\hline floralwhite [386] & 0.4 & $(0.1)$ & 0.31 & $(0.2)$ & -0.13 & $(0.6)$ & $-0.58(0.02)$ & -0.4 & $(0.1)$ & \\
\hline cyan [2911] & -0.14 & (0.6) & 0.55 & $(0.03)$ & 0.46 & $(0.07)$ & $-0.87(9 e-06)$ & 0.14 & (0.6) & \\
\hline orangered4 [97] & 0.26 & $(0.3)$ & 0.74 & $(0.001)$ & -0.38 & $(0.1)$ & $-0.62(0.01)$ & -0.26 & $(0.3)$ & -0 \\
\hline darkgrey [613] & 0.68 & $(0.004)$ & -0.46 & $(0.07)$ & -0.61 & $(0.01)$ & $0.39(0.1)$ & -0.68 & $(0.004)$ & \\
\hline darkturquoise [292] & 0.71 & $(0.002)$ & -0.8 & $(2 e-04)$ & -0.063 & $(0.8)$ & $0.15(0.6)$ & -0.71 & $(0.002)$ & \\
\hline paleturquoise [242] & 0.17 & $(0.5)$ & 0.14 & $(0.6)$ & -0.52 & $(0.04)$ & $0.21(0.4)$ & -0.17 & $(0.5)$ & -0.5 \\
\hline darkorange2 [58] & 0.36 & $(0.2)$ & -0.19 & $(0.5)$ & -0.16 & $(0.6)$ & $-0.0052(1)$ & -0.36 & $(0.2)$ & \\
\hline sienna3 [114] & -0.043 & $(0.9)$ & 0.22 & $(0.4)$ & -0.3 & $(0.3)$ & $0.12(0.7)$ & 0.043 & $(0.9)$ & \\
\hline grey [2] & 0.04 & $(0.9)$ & -0.094 & $(0.7)$ & 0.1 & $(0.7)$ & $-0.051(0.9)$ & -0.04 & (0.9) & \\
\hline (b) & Cochlearia & groenlanc & & & & & & & & \\
\hline $\begin{array}{l}\text { lightcoral [202] } \\
\text { salmon2 [169] }\end{array}$ & $\begin{array}{l}-0.22 \\
-0.24\end{array}$ & $\begin{array}{l}(0.4) \\
(0.4)\end{array}$ & $\begin{array}{l}-0.52 \\
-0.25\end{array}$ & $\begin{array}{l}(0.04) \\
(0.4)\end{array}$ & $\begin{array}{r}0.2 \\
-0.22\end{array}$ & $\begin{array}{l}(0.5) \\
(0.4)\end{array}$ & $\begin{aligned} 0.54 & (0.03) \\
0.7 & (0.002)\end{aligned}$ & $\begin{array}{l}0.22 \\
0.24\end{array}$ & $\begin{array}{l}(0.4) \\
(0.4)\end{array}$ & -1 \\
\hline darkolivegreen4 [1057] & -0.75 & $(9 e-04)$ & 0.19 & $(0.5)$ & -0.069 & $(0.8)$ & $0.62(0.01)$ & 0.75 & $(9 e-04)$ & \\
\hline thistle2 [3816] & -0.5 & $(0.05)$ & -0.33 & $(0.2)$ & -0.13 & $(0.6)$ & $0.96(7 e-09)$ & 0.5 & $(0.05)$ & \\
\hline darkred [243] & -0.3 & $(0.3)$ & 0.3 & $(0.3)$ & 0.32 & $(0.2)$ & $-0.32(0.2)$ & 0.3 & $(0.3)$ & \\
\hline brown2 [331] & -0.41 & $(0.1)$ & 0.7 & $(0.003)$ & 0.39 & $(0.1)$ & $-0.68(0.004)$ & 0.41 & $(0.1)$ & \\
\hline honeydew1 [182] & -0.098 & $(0.7)$ & 0.59 & $(0.02)$ & -0.093 & $(0.7)$ & $-0.4(0.1)$ & 0.098 & (0.7) & -0.5 \\
\hline lightcyan [1005] & -0.95 & $(3 e-08)$ & 0.36 & $(0.2)$ & 0.48 & $(0.06)$ & $0.1(0.7)$ & 0.95 & $(3 e-08)$ & \\
\hline darkgrey [227] & -0.41 & $(0.1)$ & 0.8 & $(2 e-04)$ & -0.32 & $(0.2)$ & $-0.071(0.8)$ & 0.41 & $(0.1)$ & \\
\hline royalblue [248] & -0.54 & $(0.03)$ & 0.59 & $(0.02)$ & -0.37 & $(0.2)$ & $0.32(0.2)$ & 0.54 & $(0.03)$ & \\
\hline greenyellow [342] & 0.84 & $(4 e-05)$ & -0.52 & $(0.04)$ & -0.52 & $(0.04)$ & $0.2(0.5)$ & -0.84 & $(4 e-05)$ & \\
\hline darkgreen [372] & 0.63 & $(0.009)$ & -0.28 & $(0.3)$ & -0.27 & $(0.3)$ & $-0.08(0.8)$ & -0.63 & $(0.009)$ & -0 \\
\hline plum2 [939] & 0.5 & $(0.05)$ & -0.034 & $4(0.9)$ & 0.015 & (1) & $-0.49(0.06)$ & -0.5 & $(0.05)$ & \\
\hline yellowgreen [151] & 0.22 & $(0.4)$ & 0.29 & $(0.3)$ & -0.25 & $(0.4)$ & $-0.26(0.3)$ & -0.22 & $(0.4)$ & \\
\hline magenta [4586] & 0.75 & $(9 e-04)$ & 0.16 & $(0.5)$ & -0.057 & $(0.8)$ & $-0.85(3 e-05)$ & -0.75 & $(9 e-04)$ & \\
\hline paleturquoise [402] & 0.68 & $(0.004)$ & -0.44 & $(0.09)$ & 0.24 & $(0.4)$ & $-0.49(0.05)$ & -0.68 & $(0.004)$ & \\
\hline firebrick 4 [102] & -0.42 & $(0.1)$ & 0.14 & $(0.6)$ & 0.53 & $(0.04)$ & $-0.25(0.4)$ & 0.42 & $(0.1)$ & -0.5 \\
\hline lightpink4 [83] & 0.067 & $(0.8)$ & -0.58 & $(0.02)$ & 0.024 & $(0.9)$ & $0.49(0.05)$ & -0.067 & $(0.8)$ & -0.5 \\
\hline skyblue [278] & -0.035 & & -0.14 & $(0.6)$ & 0.11 & $(0.7)$ & $0.071(0.8)$ & 0.035 & $(0.9)$ & \\
\hline ivory [124] & 0.13 & $(0.6)$ & 0.1 & $(0.7)$ & -0.28 & $(0.3)$ & $0.051(0.9)$ & -0.13 & $(0.6)$ & \\
\hline darkolivegreen [811] & 0.54 & $(0.03)$ & -0.2 & $(0.5)$ & 0.24 & $(0.4$ & $-0.59(0.02)$ & -0.54 & $(0.03)$ & \\
\hline blue2 [462] & -0.14 & $(0.6)$ & -0.22 & $(0.4)$ & 0.37 & $(0.2$ & -0.014 (1) & 0.14 & $(0.6)$ & -1 \\
\hline lavenderblush3 [366] & -0.19 & $(0.5)$ & 0.28 & $(0.3)$ & 0.55 & $(0.03)$ & $-0.63(0.008)$ & 0.19 & 10. & \\
\hline (c) & Draba nival & & & & & & & & & \\
\hline thistle2 [305] & 0.17 & $(0.5)$ & 0.55 & $(0.03)$ & -0.2 & $(0.4)$ & $-0.52(0.04)$ & -0.17 & $(0.5)$ & -1 \\
\hline antiquewhite4 [202] & -0.48 & $(0.06)$ & 0.4 & $(0.1)$ & -0.22 & $(0.4)$ & $0.31 \quad(0.2)$ & 0.48 & $(0.06)$ & \\
\hline violet [437] & -0.63 & $(0.009)$ & 0.66 & $(0.006)$ & 0.33 & $(0.2)$ & $-0.36(0.2)$ & 0.63 & $(0.009)$ & \\
\hline darkolivegreen [268] & -0.31 & $(0.2)$ & 0.014 & $(1)$ & 0.37 & $(0.2)$ & $-0.071(0.8)$ & 0.31 & $(0.2)$ & 0.5 \\
\hline coral1 [55] & -0.4 & $(0.1)$ & 0.16 & $(0.6)$ & 0.46 & $(0.07)$ & $-0.22(0.4)$ & 0.4 & $(0.1)$ & \\
\hline darkorange2 [939] & -0.99 & $(2 e-12)$ & 0.29 & $(0.3)$ & 0.47 & $(0.07)$ & $0.23 \quad(0.4)$ & 0.99 & $(2 e-12)$ & \\
\hline royalblue [2485] & -0.57 & $(0.02)$ & 0.009 & $(1)$ & 0.76 & $(6 e-04)$ & $-0.2(0.5)$ & 0.57 & $(0.02)$ & \\
\hline palevioletred3 [76] & -0.47 & $(0.06)$ & -0.11 & $(0.7)$ & 0.19 & $(0.5)$ & $0.39(0.1)$ & 0.47 & $(0.06)$ & \\
\hline skyblue3 [3422] & -0.64 & $(0.007)$ & -0.33 & $(0.2)$ & 0.098 & $(0.7)$ & $0.88(9 e-06)$ & 0.64 & $(0.007)$ & \\
\hline floralwhite [4003] & 0.86 & $(2 \mathrm{e}-05)$ & -0.026 & $(0.9)$ & -0.58 & $(0.02)$ & $-0.26(0.3)$ & -0.86 & $(2 \mathrm{e}-05)$ & \\
\hline lavenderblush3 [67] & 0.66 & $(0.005)$ & -0.62 & $(0.01)$ & -0.44 & $(0.09)$ & $0.4 \quad(0.1)$ & -0.66 & $(0.005)$ & \\
\hline lightcyan [1609] & 0.032 & $(0.9)$ & -0.33 & $(0.2)$ & -0.56 & $(0.02)$ & $0.86(2 e-05)$ & -0.032 & $(0.9)$ & \\
\hline darkgreen [3400] & 0.4 & $(0.1)$ & 0.31 & $(0.2)$ & 0.27 & $(0.3)$ & $-0.98(9 e-12)$ & -0.4 & $(0.1)$ & \\
\hline honeydew1 [338] & 0.37 & $(0.2)$ & 0.015 & $(1)$ & -0.051 & $(0.9)$ & $-0.34(0.2)$ & -0.37 & $(0.2)$ & \\
\hline
\end{tabular}

Figure 6. Heatmaps showing Pearson correlation between each co-expression module (module eigengenes) and temperature: $0 \mathrm{~h}, 3 \mathrm{~h}, 6 \mathrm{~h}$, and $24 \mathrm{~h}$ with cold treatment, and a binary measure of cold (cold treatment / no cold treatment). Each row corresponds to a co-expression module and each column to a temperature trait. The number of genes in each module is indicated in brackets after the module name. Cells show the correlation coefficient and corresponding p-value (in parentheses). From the top: a) Cardamine bellidifolia, b) Cochlearia groenlandica and c) Draba nivalis. 
Table 7. Gene Ontology terms that were overrepresented in the lightyellow $(C$. bellidifolia) lightcyan ( $C$. groenlandica) and darkorange2 ( $D$. nivalis) co-expression modules. These modules were the most positively correlated with the binary measure of cold in each species.

GO Identifier GO Term Name Domain

GO:0080167 response to karrikin $\quad$ BP

GO:0007623 circadian rhythm BP

GO:0009737 response to abscisic acid BP

GO:0006355 regulation of transcription, DNA-templated BP

GO:0009409 response to cold BP

GO:0009719 response to endogenous stimulus BP

GO:0009753 response to jasmonic acid BP

GO:0097305 response to alcohol BP

GO:0010017 red or far-red light signaling pathway $\quad$ BP

GO:0009414 response to water deprivation BP

GO:0009651 response to salt stress $\quad$ BP

GO:0005634 nucleus $\quad$ CC

GO:0003677 DNA binding MF

GO:0005509 calcium ion binding MF

GO:0043565 sequence-specific DNA binding MF

GO:0003700 DNA-binding transcription factor activity MF 


\section{Discussion}

\subsection{The cold response of Arctic Brassicaceae is highly species- specific}

Our main finding was that the cold response of the three Arctic species, C. bellidifolia, C. groenlandica, and $D$. nivalis, is highly species-specific. Not only were most DEGs unique for each species, but the number of DEGs shared by the three Arctic species and $A$. thaliana were higher than the number shared by the three Arctic species alone. This suggests that evolution of cold response in Arctic Brassicaceae followed independent genetic trajectories, but with some conserved components. These results are in concordance with our previous study of protein sequence evolution in the same three species, where we found very little overlap in positively selected genes among species, and also very few genes with convergent substitutions (Birkeland et al. 2020). Although we expected a higher degree of convergence in the cold induced expression profiles, the results agree well with those of a similar study of cold acclimation in the temperate grass subfamily Pooideae (Schubert et al. 2019). Schubert et al. (2019) found that phylogenetically diverse species of grasses showed widespread species-specific transcriptomic responses to low temperatures, but with some conserved aspects.

The independent evolutionary trajectories of cold response might be tied to the polygenic nature of the trait, involving thousands of genes. Highly polygenic traits have high genetic redundancy and should show less repeatable patterns of adaptation compared to traits involving only a few genes (Yeaman 2015; Yeaman et al. 2016; Barghi et al. 2019). Low levels of repeatability have also been found in other polygenic traits, such as drought tolerance. Marín-de la Rosa et al. (2019) recently showed that divergent strategies underlie drought resistance in closely related Brassicaceae species. However, there are also studies demonstrating a high degree of convergence in polygenic traits, like Yeaman et al. (2016) showing convergent local adaptation to climate in two distantly related conifers, and Yang et al. (2018) showing convergent evolution of flowering time in Capsella rubella due to independent deletions at the same locus (flowering time is controlled by $>60$ genes in A. thaliana; Anderson, Willis, and Mitchell-Olds 2011). Although it is not known what caused evolutionary repeatability in these exact examples, adaptation may end up taking the same routes in the presence of pleiotropic constraints or restricted available standing 
genetic variation (as also discussed in Gould and Stinchcombe 2017). Since, we find that cold tolerance mainly evolved independently in the three species, we can assume that such constraints have been of less importance. Following, there seem to be many ways to evolve cold tolerance, or at least many ways to build upon cold tolerance once a basis for cold tolerance has evolved.

\subsection{Conserved aspects of the Arctic Brassicaceae cold response}

Another major finding in our data was that the Arctic species seem to have more in common with the temperate relative $A$. thaliana, than they have with each other. This shared aspect of the cold response may represent conserved parts of the CBF pathway, which also have been found in other plant lineages (Jaglo et al. 2001; Shi, Ding, and Yang 2018). Shared genes included, for instance, those that were both tied to circadian regulation and regulation of freezing tolerance (i.e. COR27 and COR28; $\mathrm{X}$. Li et al. 2016). This fits well with the CBF pathway being gated by the circadian clock (Dong, Farré, and Thomashow 2011; Fowler, Cook, and Thomashow 2005). The Arctic cold response also seemed to follow similar trends as in A. thaliana, with a continuous increase in the number of DEGs in response to cold temperatures (Kilian et al. 2007; Calixto et al. 2018). This indicates that Arctic plants respond to low temperatures in a similar way as temperate plants, and that they are not completely hard-wired to tolerate low temperatures.

The transcriptomic changes also triggered sets of biological processes similar to the cold response of $A$. thaliana, and that of other plant species within and outside the Brassicaceae family (e.g. Zhao et al. 2012; Lee et al. 2013; Buti et al. 2018). First and foremost, this involved stress responses associated with low temperatures and freezing. The upregulation of genes associated with salt stress, water deprivation, and cadmium ion (a heavy metal highly toxic to plants; di Toppi and Gabbrielli 1999) could be linked to cold and freezing stress in two different ways. First, ice formation in the apoplast will draw water out of the cells and increase the concentration of salts and toxic solutes (Wisniewski and Fuller 1999; Körner 2003). This may lead to severe cell dehydration (Shi, Ding, and Yang 2018). Second, stress response pathways associated with cold have been shown to be partially homologous with those of dehydration and salt tolerance (Bartels and Souer 2003; Shamustakimova et al. 2017; Ingram and Bartels 1996). We also found that genes annotated with "response to oxidative stress" were overrepresented among the upregulated genes. This fits well 
with oxidative stress accompanying other abiotic stresses like cold, and especially high light intensity in combination with low temperatures (Heino and Palva 2004; Lütz 2010; Kilian et al. 2007).

Genes associated with the hormones abscisic acid (ABA) and ethylene were upregulated in response to cold in all species. ABA is an important hormone in plant stress signalling and has previously been shown to increase in abundance during cold acclimation (Heino and Palva 2004; Tuteja 2007). Exogenous application of ABA will even increase freezing tolerance in A. thaliana and other plants (Thomashow 1999). The hormone ethylene is similarly reported to influence freezing tolerance and regulates the $\mathrm{CBF}$ pathway itself (Kazan 2015). In most plant species, this entails a positive regulation of freezing tolerance (like e.g. in tomato and tobacco; Zhang and Huang 2010). However, in $A$. thaliana ethylene appears to also negatively regulate freezing tolerance depending on the growing conditions (Kazan 2015). This hormone's role in the Brassicaceae cold response is therefore still uncertain.

One way that plants increase their freezing tolerance is by accumulating compounds that in various ways hinder ice nucleation, or alleviate the effects of ice formation by protecting plant tissues against freezing damage (i.e. cryoprotectants; Ruelland et al. 2009). We observed that genes associated with possible cryoprotectants like flavonoids (especially flavonol) and sucrose were upregulated in response to cold in all species. Sucrose has been reported to lower the freezing point in A. thaliana (i.e. leading to supercooling and avoidance of freezing; Reyes-Díaz et al. 2006), to diminish the water potential between the apoplastic space and the cell in the face of ice formation (osmotic adjustment; Ruelland et al. 2009), and even to regulate cold acclimation itself (Rekarte-Cowie et al. 2008). Similarly, accumulation of flavonoids is associated with cold acclimation and higher freezing tolerance in $A$. thaliana (Schulz et al. 2016), and flavonol might have a role in protecting cell membranes during freezing stress (Korn et al. 2008). Finally, we also observed another compound that seems to be important in acquiring cold/freezing tolerance in all species. Karrikin is a compound that has received little attention in relation to cold stress, but a recent study suggests that karrikin inhibits germination under unfavourable conditions, and also improves plant vigour in the face of abiotic stress through regulation of redox homeostasis (Shah et al. 2020). Genes responsive to karrikin have also been documented to be important in the cold response of Chorispora bungeana (a subnival Brassicaceae; Zhao et al. 2012). The fact that 
flavonoids, sucrose and karrikin seem to have important roles in the cold response of four distantly related Brassicaceae species, highlights their importance in achieving cold tolerance.

These shared trends indicate that cold response in Brassicaceae is built upon a similar scaffold and support the claim that cold tolerance is a complex trait that is difficult to evolve (Donoghue 2008). It is highly likely that the last common ancestor of the three Arctic species had some kind of cold tolerance machinery as the major clades of the Brassicaceae are thought to have radiated in response to a colder and drier climate ( $33.9 \mathrm{Ma}$ in the Eocene-Oligocene transition; Huang et al. 2016). Perhaps the basis of the Arctic cold response could also have contributed to the Brassicaceae family's success in cold and dry environments. However, the highly species-specific cold responses found in C. bellidifolia, C. groenlandica, and D. nivalis also indicate that there is great evolutionary flexibility in cold coping strategies once there is a basis to build upon.

\subsection{The Arctic cold response - is there such a thing?}

The low degree of overlap in the cold response of Arctic Brassicaceae provokes the question if there exists anything like a true Arctic cold response. There are, however, a few shared genes and characteristics that stand out as potentially unique for the three Arctic species. For instance, among the $\sim 100$ genes shared only by the three Arctic species, there are particularly many transcription factors that potentially could have large effects. Yeaman et al. (2016) similarly found that convergent genes in adaptation to climate in distantly related conifers were enriched for transcription factors. Although we can only speculate about their function in this study, several are related to transcription factors that have important roles in cold tolerance in other plant species. Examples included e.g. $\mathrm{CBF}_{4}$ (mainly activated by drought in A. thaliana; Haake et al. 2002), $R A P 2.10$, and $R A P 2.2$. Considering the important role, the related $C B F 1-3$ has in regulating the cold response, $C B F 4$ stands out as a potential important candidate for the regulation of an Arctic cold response. Furthermore, this gene has also been found to be cold responsive in other species (Cai et al. 2019). Two other examples, $R A P 2.1 O$, and $R A P 2.2$, are related to $D E A R 1$; a transcription factor known to mediate freezing stress responses (Tsutsui et al. 2009). In addition, several transcription factors related to circadian rhythm like e.g., REVEILLE 2, PCL1 and $H_{5}$ are also among the DEGs shared by all Arctic species. One possibility is that the 
extreme Arctic light regime might have triggered the evolution of links between the $\mathrm{CBF}$ pathway and the circadian clock that are not found in temperate environments. We cannot completely rule out that certain aspects of the experimental light regime may have provoked the expression of these genes, but the interplay between the circadian clock and the CBF pathway is important for balancing freezing tolerance and plant growth (e.g. Dong, Farré, and Thomashow 2011; Shi, Ding, and Yang 2018).

Another interesting finding among the shared genes is the potential traces of a mitogen activated protein kinase (MAPK) cascade. Such cascades are known to be important in regulating the CBF pathway (Teige et al. 2004; Shi, Ding, and Yang 2018). Intriguingly, we found one MAPK (MAPK7), as well as two MAPKKKs (MAPKKK14 and MAPKKK18) that are uniquely upregulated in the three Arctic species. In A. thaliana, it has been shown that the MKK2 pathway regulates cold stress signaling (Teige et al. 2004), and that MAPK6 is involved in releasing inhibitory effects on CBF gene expression (Kim et al. 2017). Accordingly, the shared MAPK and MAPKKKs could potentially have important roles in a putative Arctic cold response.

Functionally, "spermidine biosynthetic process" stands out as having a special status in the Arctic species, although the significant overrepresentation was caused by only a few cold induced genes. One of these genes was arginine decarboxylase gene 1 ( $A D C 1$ ), which was tied to both the shared significant enrichment of "spermidine biosynthetic process" and "arginine decarboxylase activity" in Arctic species. This gene has previously been found to be involved in acquiring freezing tolerance in wild potato (Kou et al. 2018). It has been shown that cucumber plants pretreated with spermidine (a polyamine) show less decline in photosynthesis rates during chilling than non-treated plants (He, Nada, and Tachibana 2002). Maintaining photosynthesis rates during low temperatures should be especially important for Arctic plants as temperatures are low year-round, and accordingly, they also have optimum photosynthesis rates at lower temperatures than other plants (Chapin 1983). In addition, there was an overrepresentation of shared genes related to proline transport (an amino acid with a similar role in freezing tolerance; Ruelland et al. 2009), as well as some of the abovementioned cryoprotectants, like flavonoid biosynthesis and sucrose.

Finally, we also found other potential traces of adaptation to extreme Arctic temperatures. Some of the genes that we previously had found to be under positive 
selection (Birkeland et al. 2020), are upregulated in the same lineages in response to cold. This includes some of the top significantly differentially expressed genes, like e.g., COR15B in D. nivalis, a transmembrane protein in C. bellidifolia, and LEA46 in C. groenlandica. Furthermore, EMB2742, which has convergent substitutions in all three species (Birkeland et al. 2020), is significantly upregulated after $24 \mathrm{~h}$ of cold, possibly indicating that it has a unique Arctic function. As the findings in Birkeland et al. (2020) were not based on cold induced transcriptomes (just "untreated" transcriptomes), there could also be other positively selected genes among the cold induced DEGs that have not been detected. That important cold regulated genes are under positive selection, shows that the Arctic climate may have imposed strong selection pressure on the cold response of Arctic plants.

Despite a few potentially important overlaps in cold response, it is apparent that there probably is not a single, but many ways Arctic plants respond to low temperatures. Considering the polygenic nature of the trait, and that the three species likely descend from different temperate relatives, it would also be surprising if their cold response had converged into something uniquely Arctic. To truly understand what has made each of the three Arctic species successful in the extreme Arctic climate, a better understanding of gene functions in cold response in these species is needed. A good place to start could be to study the effects of shared transcription factors on cold and freezing tolerance.

\subsection{Limitations and future perspectives}

We note that there might be methodological differences between our study and the study on $A$. thaliana (Park et al. 2015) that may affect the number of DEGs considered as significant in each study. This may potentially have a small effect on the number of shared genes, both between A. thaliana and the Arctic species, and between the Arctic species alone. An important difference is, for instance, that our study is based on de novo assembled transcriptomes, which may have small inaccuracies in the delimitation of genes (e.g., isoforms of the same gene mistakenly being delineated as different genes). However, we also used a stringed filtering scheme to reduce inflated transcript numbers, and such differences should not affect the overall results.

This experiment represents one of the first snapshots of Arctic cold-induced transcriptomes, but more studies are needed to understand how Arctic plant species 
can cope with the low summer temperatures at higher latitudes. Future studies on Arctic cold tolerance could delve deeper into performance under long-term cold stress, or performance under cold stress in combination with high light (typical of Arctic environments).

\section{Acknowledgements}

We thank The Phytotron at the University of Oslo, and engineers Bjørn Langrekken, Marit Langrekken, and Ingrid Johansen for help with the technical setup for this experiment. We also thank the Speciation Clock Team, Xuyue Yang, Marian Schubert, Aelys Humphries, Lars Grønvold, William Hughes, and Marco Fracassetti for valuable input and comments. Computational analyses were performed on the Saga Cluster owned by Uninett/Sigma2, as well as the Abel Cluster owned by Uninett/Sigma2 and the University of Oslo (operated by the Department for Research Computing at USIT, the University of Oslo IT-department; http://www.hpc.uio.no/). The study was funded by the Research Council of Norway through the SpArc project (RCN 240223) awarded to C.B.

\section{References}

Abbott, Richard J., and Christian Brochmann. 2003. "History and Evolution of the Arctic Flora: In the Footsteps of Eric Hulten.” Molecular Ecology 12 (2): 299313.

Alexa, A., J. Rahnenfuhrer, and T. Lengauer. 2006. "Improved Scoring of Functional Groups from Gene Expression Data by Decorrelating GO Graph Structure.” Bioinformatics 22 (13): 1600-1607.

Anderson, Jill T., John H. Willis, and Thomas Mitchell-Olds. 2011. "Evolutionary Genetics of Plant Adaptation." Trends in Genetics 27 (7): 258-66.

Andrews, S. 2010. FastQC: A Quality Control Tool for High Throughput Sequence Data. https://www.bioinformatics.babraham.ac.uk/projects/fastqc/.

Archambault, Annie, and Martina V. Strömvik. 2011. "PR-10, Defensin and Cold Dehydrin Genes Are among Those over Expressed in Oxytropis (Fabaceae) Species Adapted to the Arctic.” Functional \& Integrative Genomics 11 (3): 497505.

Barghi, Neda, Raymond Tobler, Viola Nolte, Ana Marija Jakšić, François Mallard, Kathrin Anna Otte, Marlies Dolezal, Thomas Taus, Robert Kofler, and Christian Schlötterer. 2019. "Genetic Redundancy Fuels Polygenic Adaptation in Drosophila.” PLoS Biology 17 (2): e3000128.

Bartels, Dorothea, and Erik Souer. 2003. "Molecular Responses of Higher Plants to Dehydration." Topics in Current Genetics, 9-38.

Benjamini, Yoav, and Yosef Hochberg. 1995. "Controlling the False Discovery Rate: A Practical and Powerful Approach to Multiple Testing.” Journal of the Royal 
bioRxiv preprint doi: https://doi.org/10.1101/2021.12.04.471218; this version posted December 5 , 2021. The copyright holder for this preprint (which was not certified by peer review) is the author/funder, who has granted bioRxiv a license to display the preprint in perpetuity. It is made available under aCC-BY-NC 4.0 International license.

Statistical Society: Series B (Methodological) 57 (1): 289-300.

Billings, W. D. 1974. "Adaptations and Origins of Alpine Plants." Arctic and Alpine Research 6 (2): 129.

Billings, W. D., and H. A. Mooney. 1968. "The Ecology of Arctic and Alpine Plants." Biological Reviews 43 (4): 481-529.

Birkeland, Siri, A. Lovisa S. Gustafsson, Anne K. Brysting, Christian Brochmann, and Michael D. Nowak. 2020. "Multiple Genetic Trajectories to Extreme Abiotic Stress Adaptation in Arctic Brassicaceae.” Molecular Biology and Evolution 37 (7): 2052-68.

Bolger, Anthony M., Marc Lohse, and Bjoern Usadel. 2014. "Trimmomatic: A Flexible Trimmer for Illumina Sequence Data." Bioinformatics 30 (15): 2114-20.

Brochmann, C., and S. W. Steen. 1999. "Sex and Genes in the Flora of Svalbard Implications for Conservation Biology and Climate Change." In The Species Concept in the High North - A Panarctic Flora Initiative, edited by I. Nordal and V. Y. Razzhivin, 33-72. The Norwegian Academy of Science and Letters.

Buti, Matteo, Marianna Pasquariello, Domenico Ronga, Justyna Anna Milc, Nicola Pecchioni, Viet The Ho, Chiara Pucciariello, Pierdomenico Perata, and Enrico Francia. 2018. "Transcriptome Profiling of Short-Term Response to Chilling Stress in Tolerant and Sensitive Oryza Sativa Ssp. Japonica Seedlings." Functional \& Integrative Genomics 18 (6): 627-44.

Cai, Xiaoyan, Richard Odongo Magwanga, Yanchao Xu, Zhongli Zhou, Xingxing Wang, Yuqing Hou, Yuhong Wang, Yuanming Zhang, Fang Liu, and Kunbo Wang. 2019. "Comparative Transcriptome, Physiological and Biochemical Analyses Reveal Response Mechanism Mediated by CBF4 and ICE2 in Enhancing Cold Stress Tolerance in Gossypium Thurberi.” AoB PLANTS 11 (6). https://doi.org/10.1093/aobpla/plzo45.

Calixto, Cristiane P. G., Wenbin Guo, Allan B. James, Nikoleta A. Tzioutziou, Juan Carlos Entizne, Paige E. Panter, Heather Knight, Hugh G. Nimmo, Runxuan Zhang, and John W. S. Brown. 2018. "Rapid and Dynamic Alternative Splicing Impacts the Arabidopsis Cold Response Transcriptome." The Plant Cell 30 (7): 1424-44.

Camacho, Christiam, George Coulouris, Vahram Avagyan, Ning Ma, Jason Papadopoulos, Kevin Bealer, and Thomas L. Madden. 2009. "BLAST+: Architecture and Applications.” BMC Bioinformatics 10 (December): 421.

Carlsen, Tor, Walter Bleeker, Herbert Hurka, Reidar Elven, and Christian Brochmann. 2009. "Biogeography and Phylogeny of Cardamine (Brassicaceae)." Annals of the Missouri Botanical Garden 96 (2): 215-36.

Carlson, M. 2018. org.At.tair.db: Genome Wide Annotation for Arabidopsis (version $\mathrm{R}$ package version 3.7.0 ).

https://bioconductor.org/packages/release/data/annotation/html/org.At.tair.d b.html.

Chapin, F. S. 1983. "Direct and Indirect Effects of Temperature on Arctic Plants." Polar Biology 2 (1): 47-52.

Conway, Jake R., Alexander Lex, and Nils Gehlenborg. 2017. "UpSetR: An R Package for the Visualization of Intersecting Sets and Their Properties." Bioinformatics 33 (18): 2938-40.

Dong, Malia A., Eva M. Farré, and Michael F. Thomashow. 2011. "Circadian ClockAssociated 1 and Late Elongated Hypocotyl Regulate Expression of the C-Repeat Binding Factor (CBF) Pathway in Arabidopsis." Proceedings of the National Academy of Sciences of the United States of America 108 (17): 7241-46.

Donoghue, Michael J. 2008. "A Phylogenetic Perspective on the Distribution of Plant 
bioRxiv preprint doi: https://doi.org/10.1101/2021.12.04.471218; this version posted December 5 , 2021. The copyright holder for this preprint (which was not certified by peer review) is the author/funder, who has granted bioRxiv a license to display the preprint in perpetuity. It is made available under aCC-BY-NC 4.0 International license.

Diversity." Proceedings of the National Academy of Sciences of the United States of America 105 Suppl 1 (August): 11549-55.

Elvebakk, Arve. 1999. "Bioclimatic Delimitation and Subdivision of the Arctic." In The Species Concept in the High North: A Panarctic Flora Initiative, edited by Inger Nordal and V. Y. Razzhivin, 81-112. The Norwegian Academy of Science and Letters.

Elven, R., D. F. Murray, V. Y. Razzhivin, and B. A. Yurtsev. 2011. "Annotated Checklist of the Panarctic Flora (PAF). Vascular Plants.” 2011. http://panarcticflora.org/.

Emms, David M., and Steven Kelly. 2019. "OrthoFinder: Phylogenetic Orthology Inference for Comparative Genomics.” Genome Biology 20 (1): 238.

Ewels, Philip, Måns Magnusson, Sverker Lundin, and Max Käller. 2016. "MultiQC: Summarize Analysis Results for Multiple Tools and Samples in a Single Report." Bioinformatics 32 (19): 3047-48.

Fowler, Sarah G., Daniel Cook, and Michael F. Thomashow. 2005. "Low Temperature Induction of Arabidopsis CBF1, 2, and 3 Is Gated by the Circadian Clock." Plant Physiology 137 (3): 961-68.

Gentleman, Robert C., Vincent J. Carey, Douglas M. Bates, Ben Bolstad, Marcel Dettling, Sandrine Dudoit, Byron Ellis, et al. 2004. "Bioconductor: Open Software Development for Computational Biology and Bioinformatics." Genome Biology 5 (10): R80.

Gompel, Nicolas, and Benjamin Prud'homme. 2009. "The Causes of Repeated Genetic Evolution.” Developmental Biology 332 (1): 36-47.

Gould, Billie A., and John R. Stinchcombe. 2017. "Population Genomic Scans Suggest Novel Genes Underlie Convergent Flowering Time Evolution in the Introduced Range of Arabidopsis Thaliana.” Molecular Ecology 26 (1): 92-106.

Grabherr, Manfred G., Brian J. Haas, Moran Yassour, Joshua Z. Levin, Dawn A. Thompson, Ido Amit, Xian Adiconis, et al. 2011. "Full-Length Transcriptome Assembly from RNA-Seq Data without a Reference Genome." Nature Biotechnology 29 (7): 644-52.

Haake, Volker, Daniel Cook, Joséluis Riechmann, Omaira Pineda, Michael F. Thomashow, and James Z. Zhang. 2002. "Transcription Factor CBF4 Is a Regulator of Drought Adaptation in Arabidopsis.” Plant Physiology 130 (2): $639-48$.

Hart, Alexander J., Samuel Ginzburg, Muyang Sam Xu, Cera R. Fisher, Nasim Rahmatpour, Jeffry B. Mitton, Robin Paul, and Jill L. Wegrzyn. 2020. "EnTAP: Bringing Faster and Smarter Functional Annotation to Non-Model Eukaryotic Transcriptomes." Molecular Ecology Resources 20 (2): 591-604.

Heino, Pekka, and E. Tapio Palva. 2004. "Signal Transduction in Plant Cold Acclimation.” In Plant Responses to Abiotic Stress, edited by Heribert Hirt and Kazuo Shinozaki, 151-86.

He, Lixiong, Kazuyoshi Nada, and Shoji Tachibana. 2002. "Effects of Spermidine Pretreatment through the Roots on Growth and Photosynthesis of Chilled Cucumber Plants (Cucumis Sativus L.).” Engei Gakkai Zasshi. https://doi.org/10.2503/jjshs.71.490.

Huang, Chien-Hsun, Renran Sun, Yi Hu, Liping Zeng, Ning Zhang, Liming Cai, Qiang Zhang, et al. 2016. "Resolution of Brassicaceae Phylogeny Using Nuclear Genes Uncovers Nested Radiations and Supports Convergent Morphological Evolution." Molecular Biology and Evolution 33 (2): 394-412.

Ingram, J., and D. Bartels. 1996. "The Molecular Basis of Dehydration Tolerance in Plants." Annual Review of Plant Physiology and Plant Molecular Biology 47 (1): 
bioRxiv preprint doi: https://doi.org/10.1101/2021.12.04.471218; this version posted December 5 , 2021. The copyright holder for this

$377-403$.

Jaglo, Kirsten R., Susanne Kleff, Keenan L. Amundsen, Xin Zhang, Volker Haake, James Z. Zhang, Thomas Deits, and Michael F. Thomashow. 2001. "Components of the Arabidopsis C-Repeat/Dehydration-Responsive Element Binding Factor Cold-Response Pathway Are Conserved inBrassica Napus and Other Plant Species." Plant Physiology 127 (3): 910-17.

Jónsdóttir, Ingibjörg S. 2005. "Terrestrial Ecosystems on Svalbard: Heterogeneity, Complexity and Fragility from an Arctic Island Perspective.” Biology \&

Environment: Proceedings of the Royal Irish Academy 105 (3): 155-65.

Jordon-Thaden, Ingrid, Irina Hase, Ihsan Al-Shehbaz, and Marcus A. Koch. 2010.

"Molecular Phylogeny and Systematics of the Genus Draba (Brassicaceae) and Identification of Its Most Closely Related Genera." Molecular Phylogenetics and Evolution 55 (2): 524-40.

Kazan, Kemal. 2015. "Diverse Roles of Jasmonates and Ethylene in Abiotic Stress Tolerance." Trends in Plant Science 20 (4): 219-29.

Kilian, Joachim, Dion Whitehead, Jakub Horak, Dierk Wanke, Stefan Weinl, Oliver Batistic, Cecilia D’Angelo, Erich Bornberg-Bauer, Jörg Kudla, and Klaus Harter. 2007. “The AtGenExpress Global Stress Expression Data Set: Protocols, Evaluation and Model Data Analysis of UV-B Light, Drought and Cold Stress Responses." The Plant Journal: For Cell and Molecular Biology 50 (2): 347-63.

Kim, Sun Ho, Ho Soo Kim, Sunghwa Bahk, Jonguk An, Yeji Yoo, Jae-Yean Kim, and Woo Sik Chung. 2017. "Phosphorylation of the Transcriptional Repressor MYB15 by Mitogen-Activated Protein Kinase 6 Is Required for Freezing Tolerance in Arabidopsis." Nucleic Acids Research 45 (11): 6613-27.

Koch, Marcus A. 2012. "Mid-Miocene Divergence of Ionopsidium and Cochlearia and Its Impact on the Systematics and Biogeography of the Tribe Cochlearieae (Brassicaceae)." Taxon 61 (1): 76-92.

Kolde, Raivo. 2019. Pheatmap: Pretty Heatmaps (version R package version 1.0.12). https://CRAN.R-project.org/package=pheatmap.

Körner, Christian. 2003. Alpine Plant Life. Functional Plant Ecology of High Mountain Ecosystems. Springer.

Korn, Marina, Silke Peterek, Hans-Peter Mock, Arnd G. Heyer, and Dirk K. Hincha. 2008. "Heterosis in the Freezing Tolerance, and Sugar and Flavonoid Contents of Crosses between Arabidopsis Thaliana Accessions of Widely Varying Freezing Tolerance." Plant, Cell \& Environment 31 (6): 813-27.

Kou, Shuang, Lin Chen, Wei Tu, Federico Scossa, Yamei Wang, Jun Liu, Alisdair R. Fernie, Botao Song, and Conghua Xie. 2018. "The Arginine Decarboxylase Gene ADC1, Associated to the Putrescine Pathway, Plays an Important Role in Potato Cold-Acclimated Freezing Tolerance as Revealed by Transcriptome and Metabolome Analyses." The Plant Journal: For Cell and Molecular Biology 96 (6): 1283-98.

Kreps, Joel A., Yajun Wu, Hur-Song Chang, Tong Zhu, Xun Wang, and Jeff F. Harper. 2002. "Transcriptome Changes for Arabidopsis in Response to Salt, Osmotic, and Cold Stress." Plant Physiology 130 (4): 2129-41.

Lancaster, Lesley T., and Aelys M. Humphreys. 2020. "Global Variation in the Thermal Tolerances of Plants." Proceedings of the National Academy of Sciences of the United States of America. https://doi.org/10.1073/pnas.1918162117.

Langmead, Ben, and Steven L. Salzberg. 2012. "Fast Gapped-Read Alignment with Bowtie 2." Nature Methods 9 (4): 357-59.

Lee, Jungeun, Eun Kyeung Noh, Hyung-Seok Choi, Seung Chul Shin, Hyun Park, and Hyoungseok Lee. 2013. "Transcriptome Sequencing of the Antarctic Vascular 
bioRxiv preprint doi: https://doi.org/10.1101/2021.12.04.471218; this version posted December 5, 2021. The copyright holder for this preprint (which was not certified by peer review) is the author/funder, who has granted bioRxiv a license to display the preprint in perpetuity. It is made available under aCC-BY-NC 4.0 International license.

Plant Deschampsia Antarctica Desv. under Abiotic Stress.” Planta 237 (3): 82336.

Li, Bo, and Colin N. Dewey. 2011. "RSEM: Accurate Transcript Quantification from RNA-Seq Data with or without a Reference Genome.” BMC Bioinformatics 12 (1). https://doi.org/10.1186/1471-2105-12-323.

$\mathrm{Li}, \mathrm{Xu}$, Dingbang Ma, Sheen X. Lu, Xinyi Hu, Rongfeng Huang, Tong Liang, Tongda $\mathrm{Xu}$, Elaine M. Tobin, and Hongtao Liu. 2016. "Blue Light- and Low Temperature-Regulated COR27 and COR28 Play Roles in the Arabidopsis Circadian Clock." The Plant Cell 28 (11): 2755-69.

Love, Michael I., Wolfgang Huber, and Simon Anders. 2014. "Moderated Estimation of Fold Change and Dispersion for RNA-Seq Data with DESeq2." Genome Biology 15 (12): 550.

Lütz, Cornelius. 2010. "Cell Physiology of Plants Growing in Cold Environments." Protoplasma 244 (1-4): 53-73.

Marín-de la Rosa, Nora, Chung-Wen Lin, Yang Jae Kang, Stijn Dhondt, Nathalie Gonzalez, Dirk Inzé, and Pascal Falter-Braun. 2019. "Drought Resistance Is Mediated by Divergent Strategies in Closely Related Brassicaceae." The New Phytologist 223 (2): 783-97.

Pai, Athma A., and Yoav Gilad. 2014. "Comparative Studies of Gene Regulatory Mechanisms." Current Opinion in Genetics \& Development 29: 68-74.

Park, Sunchung, Chin-Mei Lee, Colleen J. Doherty, Sarah J. Gilmour, Yongsig Kim, and Michael F. Thomashow. 2015. "Regulation of the Arabidopsis CBF Regulon by a Complex Low-Temperature Regulatory Network." The Plant Journal: For Cell and Molecular Biology 82 (2): 193-207.

Patro, Rob, Geet Duggal, Michael I. Love, Rafael A. Irizarry, and Carl Kingsford. 2017. "Salmon Provides Fast and Bias-Aware Quantification of Transcript Expression." Nature Methods 14 (4): 417-19.

Rekarte-Cowie, Iona, Omar S. Ebshish, Khalifa S. Mohamed, and Roger S. Pearce. 2008. "Sucrose Helps Regulate Cold Acclimation of Arabidopsis Thaliana." Journal of Experimental Botany 59 (15): 4205-17.

Reyes-Díaz, Marjorie, Nancy Ulloa, Alejandra Zúñiga-Feest, Ana Gutiérrez, Manuel Gidekel, Miren Alberdi, Luis J. Corcuera, and León A. Bravo. 2006. “Arabidopsis Thaliana Avoids Freezing by Supercooling.” Journal of Experimental Botany 57 (14): 3687-96.

Romero, Irene Gallego, Ilya Ruvinsky, and Yoav Gilad. 2012. "Comparative Studies of Gene Expression and the Evolution of Gene Regulation.” Nature Reviews Genetics 13 (7): 505-16.

Ruelland, Eric, Marie-Noelle Vaultier, Alain Zachowski, and Vaughan Hurry. 2009. "Cold Signalling and Cold Acclimation in Plants." In Advances in Botanical Research: Volume 49, edited by Jean-Claude Kader and Michel Delseny, 35-150. Sackton, Timothy B., Phil Grayson, Alison Cloutier, Zhirui Hu, Jun S. Liu, Nicole E. Wheeler, Paul P. Gardner, et al. 2019. "Convergent Regulatory Evolution and Loss of Flight in Paleognathous Birds.” Science 364 (6435): 74-78.

Schubert, Marian, Lars Grønvold, Simen R. Sandve, Torgeir R. Hvidsten, and Siri Fjellheim. 2019. "Evolution of Cold Acclimation and Its Role in Niche Transition in the Temperate Grass Subfamily Pooideae." Plant Physiology 180 (1): 404-19.

Schulz, Elisa, Takayuki Tohge, Ellen Zuther, Alisdair R. Fernie, and Dirk K. Hincha. 2016. "Flavonoids Are Determinants of Freezing Tolerance and Cold Acclimation in Arabidopsis Thaliana." Scientific Reports 6 (September): 34027.

Shah, Faheem Afzal, Xiao Wei, Qiaojian Wang, Wenbo Liu, Dongdong Wang, Yuanyuan Yao, Hao Hu, et al. 2020. "Karrikin Improves Osmotic and Salt Stress 
bioRxiv preprint doi: https://doi.org/10.1101/2021.12.04.471218; this version posted December 5 , 2021. The copyright holder for this preprint (which was not certified by peer review) is the author/funder, who has granted bioRxiv a license to display the preprint in perpetuity. It is made available under aCC-BY-NC 4.0 International license.

Tolerance via the Regulation of the Redox Homeostasis in the Oil Plant Sapium Sebiferum." Frontiers in Plant Science 11.

https://doi.org/10.3389/fpls.2020.00216.

Shamustakimova, A. O., T. G. Leonova, V. V. Taranov, A. H. de Boer, and A. V. Babakov. 2017. "Cold Stress Increases Salt Tolerance of the Extremophytes Eutrema Salsugineum (Thellungiella Salsuginea) and Eutrema (Thellungiella) Botschantzevii." Journal of Plant Physiology 208 (January): 128-38.

Shi, Yiting, Yanglin Ding, and Shuhua Yang. 2018. "Molecular Regulation of CBF Signaling in Cold Acclimation." Trends in Plant Science 23 (7): 623-37.

Simão, Felipe A., Robert M. Waterhouse, Panagiotis Ioannidis, Evgenia V. Kriventseva, and Evgeny M. Zdobnov. 2015. "BUSCO: Assessing Genome Assembly and Annotation Completeness with Single-Copy Orthologs." Bioinformatics 31 (19): 3210-12.

Steponkus, P. L. 1984. "Role of the Plasma Membrane in Freezing Injury and Cold Acclimation." Annual Review of Plant Physiology 35 (1): 543-84.

Stern, David L. 2013. “The Genetic Causes of Convergent Evolution.” Nature Reviews Genetics 14 (11): 751-64.

Teige, Markus, Elisabeth Scheikl, Thomas Eulgem, Róbert Dóczi, Kazuya Ichimura, Kazuo Shinozaki, Jeffery L. Dangl, and Heribert Hirt. 2004. "The MKK2 Pathway Mediates Cold and Salt Stress Signaling in Arabidopsis.” Molecular Cell 15 (1): 141-52.

Thomashow, Michael F. 1999. "Plant Cold Acclimation: Freezing Tolerance Genes and Regulatory Mechanisms.” Annual Review of Plant Physiology and Plant Molecular Biology 50 (June): 571-99.

- - . 2010. "Molecular Basis of Plant Cold Acclimation: Insights Gained from Studying the CBF Cold Response Pathway." Plant Physiology 154 (2): 571-77.

Toppi, L. Sanità di, L. Sanità di Toppi, and R. Gabbrielli. 1999. "Response to Cadmium in Higher Plants." Environmental and Experimental Botany 41 (2): 105-30.

Tsutsui, Tomokazu, Wataru Kato, Yutaka Asada, Kaori Sako, Takeo Sato, Yutaka Sonoda, Satoshi Kidokoro, et al. 2009. "DEAR1, a Transcriptional Repressor of DREB Protein That Mediates Plant Defense and Freezing Stress Responses in Arabidopsis.” Journal of Plant Research 122 (6): 633-43.

Tuteja, Narendra. 2007. "Abscisic Acid and Abiotic Stress Signaling." Plant Signaling \& Behavior 2 (3): 135-38.

Wang, Jiangshan, Quan Zhang, Feng Cui, Lei Hou, Shuzhen Zhao, Han Xia, Jingjing Qiu, et al. 2017. "Genome-Wide Analysis of Gene Expression Provides New Insights into Cold Responses in Thellungiella Salsuginea." Frontiers in Plant Science 8. https://doi.org/10.3389/fpls.2017.00713.

Wang, Minghui, Yongzhong Zhao, and Bin Zhang. 2015. "Efficient Test and Visualization of Multi-Set Intersections.” Scientific Reports 5 (November): 16923.

Wiens, John J., and Michael J. Donoghue. 2004. "Historical Biogeography, Ecology and Species Richness." Trends in Ecology \& Evolution 19 (12): 639-44.

Wisniewski, M., and M. Fuller. 1999. "Ice Nucleation and Deep Supercooling in Plants: New Insights Using Infrared Thermography.” In Cold Adapted Organisms, edited by R. Margesin and F. Schinner, 105-18. Springer.

Wisniewski, M., M. Fuller, J. Palta, J. Carter, and R. Arora. 2004. "Ice Nucleation, Propagation, and Deep Supercooling in Woody Plants." Journal of Crop Improvement 10 (1-2): 5-16.

Yang, Li, Hui-Na Wang, Xing-Hui Hou, Yu-Pan Zou, Ting-Shen Han, Xiao-Min Niu, 
Jie Zhang, et al. 2018. "Parallel Evolution of Common Allelic Variants Confers Flowering Diversity in Capsella Rubella." The Plant Cell 30 (6): 1322-36.

Yeaman, Sam. 2015. "Local Adaptation by Alleles of Small Effect." The American Naturalist 186 Suppl 1 (October): S74-89.

Yeaman, Sam, Kathryn A. Hodgins, Katie E. Lotterhos, Haktan Suren, Simon

Nadeau, Jon C. Degner, Kristin A. Nurkowski, et al. 2016. "Convergent Local

Adaptation to Climate in Distantly Related Conifers.” Science 353 (6306): 143133 .

Zhang, Zhijin, and Rongfeng Huang. 2010. "Enhanced Tolerance to Freezing in

Tobacco and Tomato Overexpressing Transcription Factor TERF2/LeERF2 Is

Modulated by Ethylene Biosynthesis." Plant Molecular Biology 73 (3): 241-49.

Zhao, Zhiguang, Lingling Tan, Chunyan Dang, Hua Zhang, Qingbai Wu, and Lizhe

An. 2012. "Deep-Sequencing Transcriptome Analysis of Chilling Tolerance Mechanisms of a Subnival Alpine Plant, Chorispora Bungeana." BMC Plant Biology 12 (1): 222. 